Article

\title{
Synthesis, characterization, and catalytic performance of hierarchical ZSM-11 zeolite synthesized via dual-template route
}

\author{
Hui Liu a,b,*, Shuang Zhang a, Sujuan Xie a, Wanshuo Zhang a,c, Wenjie Xin a, Shenglin Liu a, \\ Longya $\mathrm{Xu}$ a,\# \\ a Dalian National Laboratory for Clean Energy, Dalian Institute of Chemical Physics, Chinese Academy of Sciences, Dalian 116023, Liaoning, China \\ ${ }^{\mathrm{b}}$ Research and Development Center, Sinochem Quanzhou Petrochemical Co., Ltd, Quanzhou 362103, Fujian, China \\ c College of Chemistry and Chemical Engineering, Harbin Normal University, Harbin 150025, Heilongjiang, China
}

\section{A R T I C L E I N F O}

\section{Article history:}

Received 5 October 2017

Accepted 21 November 2017

Published 5 January 2018

\section{Keywords:}

Hierarchical material

ZSM-11

Dual-template

Cetyltrimethylammonium bromide

Alkylation of benzene

\begin{abstract}
A B S T R A C T
Hierarchical zeolite materials were prepared via one-pot synthesis of ZSM-11 zeolites with different molar ratios $(R)$ of a mesoporogen, i.e., cetyltrimethylammonium bromide template (CTAB), to a microporogen, i.e., tetra- $n$-butylammonium bromide (TBABr). The structures, morphologies, and textural properties of the resultant materials were investigated. Initially, with increasing $R$, the crystal size of the synthesized product decreased, the number of intercrystalline mesopores increased, and a pure ZSM-11 zeolite phase was present. Then an MCM-41-like phase was produced and embedded in the ZSM-11 zeolite phase. Finally, an MCM-41-like phase was obtained. The alkalinity had important effects on the physicochemical and textural properties of the prepared samples. A possible mechanism of formation of the hierarchical ZSM-11 zeolite was proposed on the basis of a combination of various characterization results. The role of CTAB varied depending on the $R$ value, and it showed a capping effect, micellar effect, and template effect. These effects of CTAB were synergetic in ZSM-11 synthesis, but they were competitive with the structure-directing effect of TBABr. In addition, the impact of the acidic properties and porosities of the hierarchical ZSM-11 catalysts on their performances in the alkylation of benzene with dimethyl ether was investigated.
\end{abstract}

(C) 2018, Dalian Institute of Chemical Physics, Chinese Academy of Sciences. Published by Elsevier B.V. All rights reserved.

\section{Introduction}

ZSM-11 zeolites, which have two crossing straight channels $(0.53 \mathrm{~nm} \times 0.54 \mathrm{~nm}, 0.53 \mathrm{~nm} \times 0.54 \mathrm{~nm})$, and are topologically similar to ZSM-5, have received much attention because of their excellent performances as solid acid and shape-selective catalysts in various industrial processes [1,2]. Zeolite catalysts such as ZSM-11 have excellent properties, e.g., a high surface area, high hydrothermal stability, intrinsic acidity, well-defined microporosity, and the ability to confine active species $[3,4]$. However, despite these superior features, this zeolite only has small micropores, and this significantly inhibits the mass transfer of reactants and products to/from the active sites, which results in relatively easy coke formation and catalyst deactivation $[5,6]$. One strategy for overcoming these problems is to introduce substantial intra- or inter-crystalline mesopores in addition to the microporous structure, leading to the creation of a hierarchical zeolite. In particular, mesopore introduction into the zeolite crystals to give ZSM-11 with a hierarchical architecture can lead to superior performances in various reactions [7-9].

Intensive efforts have been devoted to preparing hierar-

\footnotetext{
* Corresponding author. Tel/Fax: +86-595-27570458; E-mail: liuhui22@sinochem.com

\# Corresponding author. Tel/Fax: +86-411-84379279; E-mail: lyxu@dicp.ac.cn

DOI: 10.1016/S1872-2067(17)62984-X | http://www.sciencedirect.com/science/journal/18722067 | Chin. J. Catal., Vol. 39, No. 1, January 2018
} 
chical zeolites and a variety of synthetic methods have been developed, such as chemical leaching (desilication and dealumination) [10,11], assembly of nanosized zeolite crystals $[12,13]$, and synthesis in the presence of various hard or soft templates [14,15], and have mainly focused on ZSM-5. In recent years, the dual-templating method, which involves use of a template for microporosity and a supramolecular soft template such as a cationic surfactant for generation of mesoporosity, has been drawing increasing attention because of its good compatibility with zeolite precursors and high efficiency in creating mesoporosity [16,17].

The soft templates can be organosilanes, cationic polymers, silylated polymers, general surfactants, and other agents with unique molecular structures. Organosilane soft templates consisting of specially designed bifunctional coupling agents can prevent phase separation of the amorphous mesoporous material and bulky zeolite crystals during crystallization [18]. Cationic polymers have been used as mesoporogens in the synthesis of hierarchical beta and ZSM-5 zeolites [19]. Silylated polypropylene oxide diamine, polyethylenimine polymers [20], and the bifunctionalized agent $\mathrm{C}_{22-6-6}$ [21] have been successfully used in the synthesis of hierarchical zeolites. However, despite extensive research, the synthesis of these unique templates is complex, therefore the preparation of mesoporous zeolite materials is expensive [22].

The use of the common surfactant cetyltrimethylammonium bromide (CTAB) to prepare hierarchical zeolites has attracted great interest since the development of MCM-41. In recent years, there have been many reports of CTAB-assisted synthesis of hierarchical zeolites. For example, hierarchical ZSM-5 and Y zeolites have been prepared using CTAB, and their mesoporous structures can be tuned by the addition of the swelling agent 1,3,5-trimethylbenzene [23,24]. However, phase separation in the synthetic system is hard to avoid when CTAB is used as a mesoporogen, mainly because of weak binding between CTAB and silicate species [25]. Methods for solving this problem have been explored, such as microwave-induced assembly via ionic interactions between sulfonic acid-functionalized nanozeolites and counter cationic surfactants [26,27], and adjusting the $\mathrm{pH}$ value of the synthetic solution [25]. It was reported that microporous and mesoporous phases were formed with the assistance of structure-directing agents and surfactants, respectively [23]. However, the effects of the relative amounts of these agents on the resultant samples and their specific roles have not been studied in detail.

Adjustment of the $\mathrm{pH}$ during treatment of a zeolite with CTAB under alkaline conditions clearly affects the physicochemical and textural properties of the final products [28-30]. Hierarchical zeolites with intracrystalline mesopores or zeolite/MCM-41 composites can be obtained by adjusting the $\mathrm{pH}$ of the mixture. For an ethanol/water mixed solution containing both micro- and meso-templates (tetra- $n$-butylammonium hydroxide and $\mathrm{CTAB}$ ), $\mathrm{pH}$ adjustment affects the interactions among various species in the mixture and results in the formation of completely different species [25].

Benzene alkylation, e.g., with methanol, ethanol, ethylene, and propylene, is an effective method for benzene reduction
$[31,32]$. The use of dimethyl ether (DME) has also attracted much attention because it is green, nontoxic, and readily available [33]. Our previous work showed that benzene alkylation with DME over Zn-loaded [34], aluminophosphate-bound [35], alkali-treated [8], and surfactant-assisted alkaline-treated [7] ZSM-11 zeolites is a highly efficient approach to the production of high-value aromatics.

In the present work, a series of hierarchical ZSM-11 zeolite materials with varying degrees of mesoporosity were prepared via a dual-template (tetra- $n$-butylammonium bromide (TBABr) and $\mathrm{CTAB}$ ) route. The mesoporosities of the samples were investigated on the basis of the correlations between the structure, texture, acidity, and catalytic performance.

\section{Experimental}

\subsection{Sample preparation}

The chemicals used for material preparation were silica sol (25.59 wt $\% \mathrm{SiO}_{2}, 0.31$ wt $\% \mathrm{Na}_{2} \mathrm{O}, 0.10$ wt $\% \mathrm{Al}_{2} \mathrm{O}_{3}, 73.99$ wt $\%$ $\mathrm{H}_{2} \mathrm{O}$, Qindao Haiyang Chemical Co., Ltd.), aluminum sulfate $\left(\mathrm{Al}_{2}\left(\mathrm{SO}_{4}\right)_{3} \cdot 18 \mathrm{H}_{2} \mathrm{O}\right.$, Tianjin Kemiou Chemical Reagent Co., Ltd.), $\mathrm{NaOH}$ (96.0 wt\%, Sinopharm Chemical Reagent Co., Ltd.), TBABr (99.0 wt\%, Tianjin Bodi Chemical Co., Ltd.), CTAB (99 wt\%, J\&K Chemical Ltd.), and deionized water.

\subsubsection{Synthesis of microporous ZSM-11}

A conventional microporous ZSM-11 zeolite was synthesized as described in the literature [1], but a slightly different molar composition, i.e., $12 \mathrm{Na}_{2} \mathrm{O}: 1.23 \mathrm{Al}_{2} \mathrm{O}_{3}: 80 \mathrm{SiO}_{2}: 4 \mathrm{TBABr}$ : $3600 \mathrm{H}_{2} \mathrm{O}$, was used. The zeolite material was crystallized under autogenous pressure at $150{ }^{\circ} \mathrm{C}$ for $48 \mathrm{~h}$; the obtained sample was denoted by NaZSM-11.

\subsubsection{Synthesis of hierarchical ZSM-11}

A hierarchical ZSM-11 zeolite with the following molar composition was synthesized: $12 \mathrm{Na}_{2} \mathrm{O}: 1.23 \mathrm{Al}_{2} \mathrm{O}_{3}: 80 \mathrm{SiO}_{2}: 4$ TBABr : (1-24) CTAB : $3600 \mathrm{H}_{2} \mathrm{O}$. In a typical run, aluminum sulfate $(0.87 \mathrm{~g})$ was dissolved in deionized water. Silica sol (17.34 g), NaOH solution (3.11 g, $31.98 \mathrm{wt} \%$ ), TBABr (1.42 g), and CTAB $(0.40 \mathrm{~g})$ were added sequentially, with constant stirring with a glass rod; the total mass of water was $56.87 \mathrm{~g}$. The mixtures were introduced into $100 \mathrm{~mL}$ stainless-steel autoclaves with a Teflon lining rotated at $100 \mathrm{rpm}$, and crystallized under autogenous pressure at $150{ }^{\circ} \mathrm{C}$ for $48 \mathrm{~h}$. The solid product was separated by centrifugation, washed several times with deionized water until the washing water was neutral, dried overnight at $120^{\circ} \mathrm{C}$, and calcined at $550{ }^{\circ} \mathrm{C}$ for $6 \mathrm{~h}$ to remove residual CTAB and TBABr. The obtained samples were denoted by NaZ-CTAB- $R$, where $R$ represents the molar ratio of CTAB to TBABr.

To investigate the effects of alkalinity on the structures and textural properties of the samples synthesized in the presence of CTAB, two more samples were prepared with the reagent molar composition $x \mathrm{Na}_{2} \mathrm{O}: 1.23 \mathrm{Al}_{2} \mathrm{O}_{3}: 80 \mathrm{SiO}_{2}: 4 \mathrm{TBABr}: 20$ CTAB : $3600 \mathrm{H}_{2} \mathrm{O}$, where $x$ was 15 or 18; the obtained samples were denoted by NaZ-CTAB-5a and NaZ-CTAB-5b, respectively. 
The synthesized Na-form zeolite samples were treated three times with $\mathrm{NH}_{4} \mathrm{NO}_{3}$ solution $\left(0.8 \mathrm{~mol} / \mathrm{L}, 80^{\circ} \mathrm{C}, 1 \mathrm{~h}, 20 \mathrm{~cm}^{3} / \mathrm{g}\right.$ of zeolite), washed, and dried to produce the $\mathrm{NH}_{4}$-form zeolites. The $\mathrm{NH}_{4}$-form zeolites were transformed into the H-forms by calcination at $510{ }^{\circ} \mathrm{C}$ for $3 \mathrm{~h}$. The obtained samples were denoted by HZ-CTAB- $R$. The synthesized H-form ZSM-11, without CTAB addition, was denoted by HZSM-11. All the samples were pressed, crushed, and sieved to $0.38-0.85 \mathrm{~mm}$ particles before they were loaded into the reactor.

\subsection{Characterization methods}

X-ray diffraction (XRD) patterns were recorded using an $\mathrm{X}^{\prime}$ Pert PRO X-ray diffractometer, with $\mathrm{Cu} K_{\alpha}$ radiation $(40 \mathrm{kV}, 40$ $\mathrm{mA}$ ), in the $2 \theta$ scanning range from $5^{\circ}$ to $50^{\circ}$ at a scanning rate of $10^{\circ} / \mathrm{min}$. The relative crystallinity (RC) was calculated based on the relative areas of the reflections at $2 \theta=7.9 \pm 0.1^{\circ}, 8.8 \pm$ $0.1^{\circ}, 23.0 \pm 0.1^{\circ}, 23.8 \pm 0.1^{\circ}$, and $45.0 \pm 0.1^{\circ}$ in the patterns of the prepared samples and the pristine ZSM-11, for which RC was assumed to be $100 \%$. X-ray fluorescence was performed using a Philips Magix 601X spectrometer.

$\mathrm{N}_{2}$ adsorption and desorption experiments were performed using a Micromeritics ASAP-2020 system at liquid $\mathrm{N}_{2}$ temperature, i.e., $-196{ }^{\circ} \mathrm{C}$. Prior to analysis, each sample was degassed at $350{ }^{\circ} \mathrm{C}$ for $10 \mathrm{~h}$. The Brunauer-Emmett-Teller (BET) equation was used to calculate the specific surface areas from the adsorption branch, and the $t$-plot method was used to estimate the microporous volumes of the zeolite materials.

Scanning electron microscopy (SEM) was performed using a JSM-7800 F field-emission microscope at an accelerating voltage of $30 \mathrm{kV}$. Transmission electron microscopy (TEM) images were obtained using a JEM-2100 microscope at an accelerating voltage of $200 \mathrm{kV}$. Prior to observations, the samples were ultrasonically dispersed in anhydrous alcohol, and the resultant suspension was added dropwise to a micro-grid membrane and dried in air.

Solid-state ${ }^{29} \mathrm{Si}$ and ${ }^{27} \mathrm{Al}$ magic-angle-spinning nuclear magnetic spectroscopies (MAS NMR) were performed using a Bruker Avance III $500 \mathrm{MHz}$ spectrometer. Prior to ${ }^{27} \mathrm{Al}$ MAS NMR measurements, all the samples were fully hydrated with $\mathrm{NH}_{4} \mathrm{NO}_{3}$ saturated solution in a closed container.

Pyridine adsorption infrared (Py-IR) spectroscopy was performed using a Vertex 70 IR spectrometer at a resolution of 4 $\mathrm{cm}^{-1}$. The sample powder was pressed into a self-supported wafer (13 mm diameter) of mass ca. $10 \mathrm{mg}$, and then the wafer was placed in an in situ cell. The fresh samples were pretreated as follows. The cell containing the zeolite wafer was evacuated while slowly increasing the temperature from room temperature (r.t.) to $450{ }^{\circ} \mathrm{C}$ and then evacuated at $450{ }^{\circ} \mathrm{C}$ for $1 \mathrm{~h}$. A background spectrum was recorded after the wafer had cooled to r.t. The sample was then saturated with pyridine vapor for $20 \mathrm{~min}$ at $0{ }^{\circ} \mathrm{C}$ and excess pyridine was removed under vacuum at $150{ }^{\circ} \mathrm{C}$ for $0.5 \mathrm{~h}$. The IR spectrum of the sample was recorded at r.t. to quantify the total acidity. Excess pyridine on the sample was removed under vacuum at $450{ }^{\circ} \mathrm{C}$ for $0.5 \mathrm{~h}$, after cooling to r.t. An IR spectrum was recorded and used to quantify the acid strength. The bands at ca. 1540 and ca. $1450 \mathrm{~cm}^{-1}$ were integrated to determine the concentrations of Brönsted (B) and Lewis (L) acid sites, respectively.

\subsection{Catalyst evaluation}

The alkylation of benzene with DME was performed in a stainless-steel fixed-bed reactor. In a typical run, the catalyst $(0.5 \mathrm{~g})$ was placed in the middle part of the reactor and pretreated at $500{ }^{\circ} \mathrm{C}$ for $1 \mathrm{~h}$ in $\mathrm{N}_{2}$ flow. Unless specified, the reaction was performed under the conditions $350{ }^{\circ} \mathrm{C}, 0.1 \mathrm{MPa}$, $n$ (benzene $) / n(\mathrm{DME})=2$, and a DME weight hourly space velocity (WHSV) of $16 \mathrm{~h}^{-1}$. Gas and liquid products were collected and analyzed using a gas chromatography system (Agilent 7890 A) equipped with a flame ionization detector and a PONA capillary column $(50 \mathrm{~m} \times 0.2 \mathrm{~mm} \times 0.5 \mu \mathrm{m})$.

\section{Results and discussion}

\subsection{Structural and physicochemical characterization}

The wide-angle XRD patterns of microporous (NaZSM-11) and hierarchical (NaZ-CTAB-R) ZSM-11 samples are shown in Fig. 1(a). The patterns of all the samples show the characteristic diffraction peaks of a MEL-type zeolite, except that of NaZ-CTAB-5.2, with a fairly weak peak, and that of NaZ-CTAB-6, with no clear peak. Although the peak intensities decreased with increasing $R$, the characteristic diffraction peaks of the MEL zeolite were preserved, even for NaZ-CTAB-5. However, a higher $R$ value caused a significant decrease in the peak intensities, indicating a noticeable loss of crystallinity. The data in Table 1 show that the RC values of the NaZ-CTAB- $R$ samples decreased with increasing $R$, compared with that of the NaZSM-11 zeolite.

The small-angle XRD patterns (Fig. 1(b)) show that although the samples with low $R$ values gave no clear peak, the peak became clearer with increasing $R$. In particular, for $R \geq 5$, an intense and broad peak was observed at ca. $2 \theta=2^{\circ}$ for the NaZ-CTAB- $R$ samples, and the intensity of this peak increased with increasing $R$ value. The results indicate that an ordered mesoporous phase was formed with the assistance of CTAB. It should be noted that this mesoporous phase was different from the long-range ordered hexagonal MCM-41 phase, with three diffraction peaks, and the peaks were clearly broader. This may be because insufficient silica was used in the sample synthesis [30]. This resulted in the production of poorly ordered mesoporous materials, which can be attributed to an MCM-41-like phase. The wide- and small-angle XRD data indicate that the NaZ-CTAB- $R$ samples with low $R$ values consisted of a pure ZSM-11 phase, whereas samples with higher $R$ values were ZSM-11/MCM-41-like composites. The decrease in RC with increasing $R$ can be ascribed to the presence of smaller crystalline domains, a larger number of defects [36], and the production of an amorphous mesoporous phase. An ordered mesoporous material shows reflections in the low-angle region only, whereas the remaining part is featureless [37]. NaZ-CTAB-6 therefore consisted almost entirely of MCM-41-like materials.

The addition of mesoporous templates to the synthesis gel 

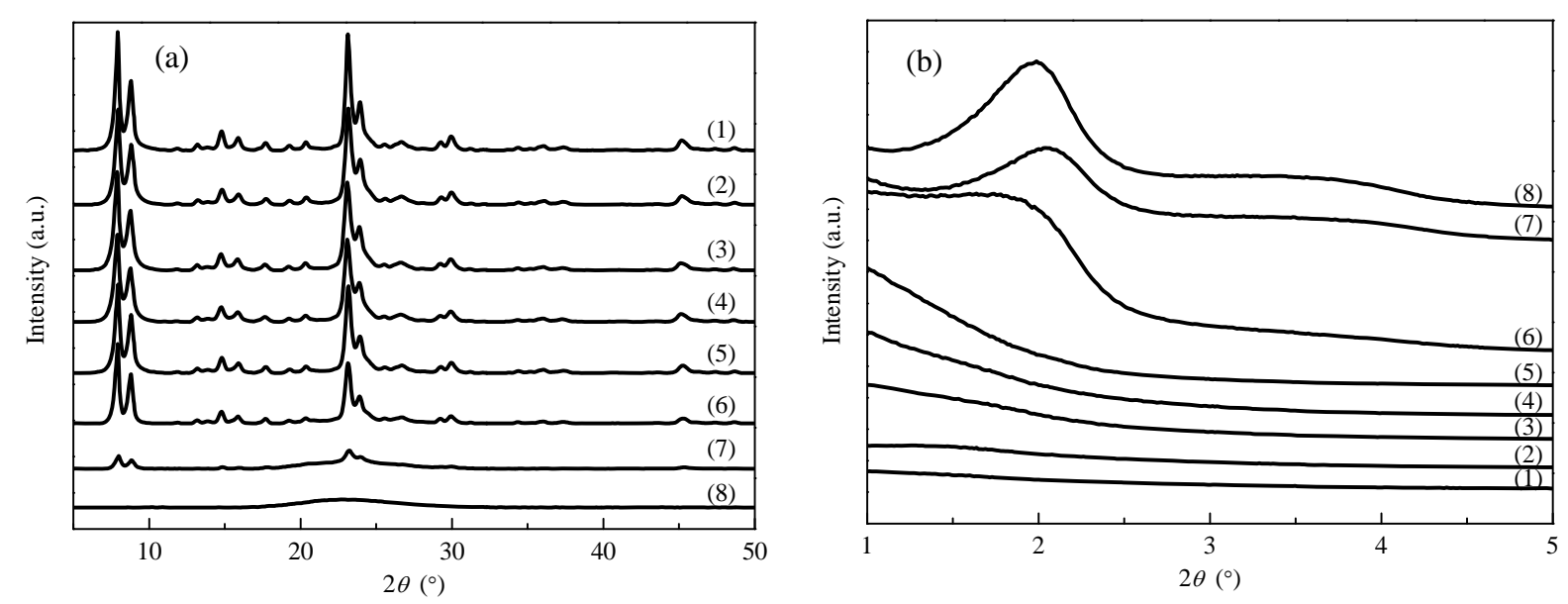

Fig. 1. Wide-angle (a) and small-angle (b) XRD patterns of (1) NaZSM-11, (2) NaZ-CTAB-0.5, (3) NaZ-CTAB-1, (4) NaZ-CTAB-3, (5) NaZ-CTAB-4, (6) NaZ-CTAB-5, (7) NaZ-CTAB-5.2, and (8) NaZ-CTAB-6.

Table 1

Textural and physiochemical data for HZSM-11 and HZ-CTAB-R samples.

\begin{tabular}{|c|c|c|c|c|c|c|c|c|c|c|c|}
\hline \multirow[b]{2}{*}{ Sample } & \multirow[b]{2}{*}{$\mathrm{Si} / \mathrm{Al}_{2}{ }^{\mathrm{b}}$} & \multirow[b]{2}{*}{$\mathrm{RC} / \%^{\mathrm{c}}$} & \multirow[b]{2}{*}{$\mathrm{HF}^{\mathrm{d}}$} & \multicolumn{4}{|c|}{ Textural data } & \multicolumn{2}{|c|}{ Conversion at $3 \mathrm{~h} \mathrm{( \% )}$} & \multicolumn{2}{|c|}{ Conversion at $30 \mathrm{~h}(\%)$} \\
\hline & & & & $\begin{array}{c}A_{\mathrm{BET}} \\
\left(\mathrm{m}^{2} / \mathrm{g}\right)\end{array}$ & $\begin{array}{c}A_{\text {ext }} \\
\left(\mathrm{m}^{2} / \mathrm{g}\right)\end{array}$ & $\begin{array}{c}V_{\text {micro }} \\
\left(\mathrm{cm}^{3} / \mathrm{g}\right)\end{array}$ & $\begin{array}{c}V_{\text {meso }} \\
\left(\mathrm{cm}^{3} / \mathrm{g}\right)\end{array}$ & DME & Benzene & DME & Benzene \\
\hline HZSM-11 & 50.6 & 100 & 0.011 & 397 & 105 & 0.132 & 0.180 & 99.59 & 38.66 & 91.57 & 23.67 \\
\hline HZ-CTAB- 0.25 a & 50.3 & 96 & 0.018 & 416 & 144 & 0.123 & 0.290 & 96.90 & 28.50 & 92.04 & 23.41 \\
\hline HZ-CTAB-0.5 & 50.6 & 89 & 0.022 & 427 & 171 & 0.118 & 0.349 & 99.98 & 40.59 & 98.52 & 36.62 \\
\hline HZ-CTAB-1 & 54.7 & 86 & 0.027 & 465 & 203 & 0.118 & 0.412 & 99.55 & 38.21 & 97.91 & 33.53 \\
\hline HZ-CTAB-1.5 & 52.0 & 85 & 0.034 & 491 & 234 & 0.116 & 0.492 & - & - & - & - \\
\hline HZ-CTAB-2 & 58.3 & 83 & 0.040 & 502 & 248 & 0.115 & 0.582 & 99.28 & 37.88 & 97.87 & 33.00 \\
\hline HZ-CTAB-3 & 64.3 & 82 & 0.039 & 501 & 251 & 0.113 & 0.577 & 97.72 & 36.96 & 94.92 & 27.72 \\
\hline HZ-CTAB-4 & 63.1 & 81 & 0.024 & 443 & 211 & 0.104 & 0.378 & 99.45 & 35.75 & 95.59 & 28.48 \\
\hline HZ-CTAB-4.3 & 53.5 & 80 & 0.025 & 445 & 216 & 0.103 & 0.394 & - & - & - & - \\
\hline HZ-CTAB-4.5 & 62.2 & 77 & 0.030 & 569 & 372 & 0.087 & 0.443 & - & - & - & - \\
\hline HZ-CTAB-5 & 61.5 & 57 & 0.029 & 565 & 369 & 0.086 & 0.438 & 97.98 & 31.05 & 92.74 & 25.83 \\
\hline HZ-CTAB-5a & 59.4 & 78 & 0.029 & 537 & 341 & 0.087 & 0.432 & 98.03 & 31.55 & 90.62 & 22.04 \\
\hline HZ-CTAB-5b & 59.2 & 95 & 0.024 & 447 & 216 & 0.104 & 0.370 & 99.41 & 37.90 & 93.88 & 27.88 \\
\hline HZ-СTAB-5.2 & 61.9 & 15 & 0.022 & 761 & 674 & 0.030 & 0.810 & - & - & - & - \\
\hline HZ-CTAB-6 & 66.4 & - & 0.014 & 857 & 795 & 0.016 & 0.938 & 57.01 & -18.31 & 39.19 & -27.38 \\
\hline
\end{tabular}

a Molar ratio of CTAB to TBABr.

${ }^{\mathrm{b}} \mathrm{Si} / \mathrm{Al}_{2}$ molar ratio determined by X-ray fluorescence.

'Relative crystallinity calculated from XRD results for $\mathrm{Na}$-form samples.

${ }^{\mathrm{d}}$ Hierarchical factor.

not only influenced the product structures and RC values, but also significantly altered the final product morphologies. The SEM images of selected samples are shown in Fig. 2. The synthesized NaZSM-11 had a spherical aggregated structure, which was self-assembled from clubbed crystalline grains of ca. 60-90 $\mathrm{nm}$ in length. When $R$ was less than 3 , the NaZ-CTAB- $R$ samples basically retained the spherical aggregated structure, but the lengths of the rod-like nanoparticles clearly decreased with increasing $R$ value. For the NaZ-CTAB-4 sample, a small amount of an amorphous phase appeared with the zeolite phase. The proportion of the amorphous phase increased with further increases in $R$. It is worth noting that the sizes of the aggregated structure and crystalline grains of NaZ-CTAB- $R$ fluctuated with increasing $R(R>4)$. When $R$ reached 6, the sample was almost amorphous. The XRD results (Fig. 1) suggest that these amorphous materials are poorly ordered mesoporous MCM-41-like phases. Our results are in good agreement with previous reports. For example, amorphous materials were also observed in SEM images of ZSM-5 synthesized in the presence of CTAB [38]. In the case of SAPO-34 synthesis, with increasing of CTAB concentration, the crystal size first decreased, and then an amorphous phase was produced [39].

The decrease in the ZSM-11 crystal size could be caused by the monovalent cationic surfactant CTAB acting as a capping agent [40], which would prevent further growth of primary nanosized zeolite crystals. However, in the case of MOR zeolite synthesis, the monovalent cationic surfactant CTAB had almost no effect on the size of the resultant zeolite [41]. 

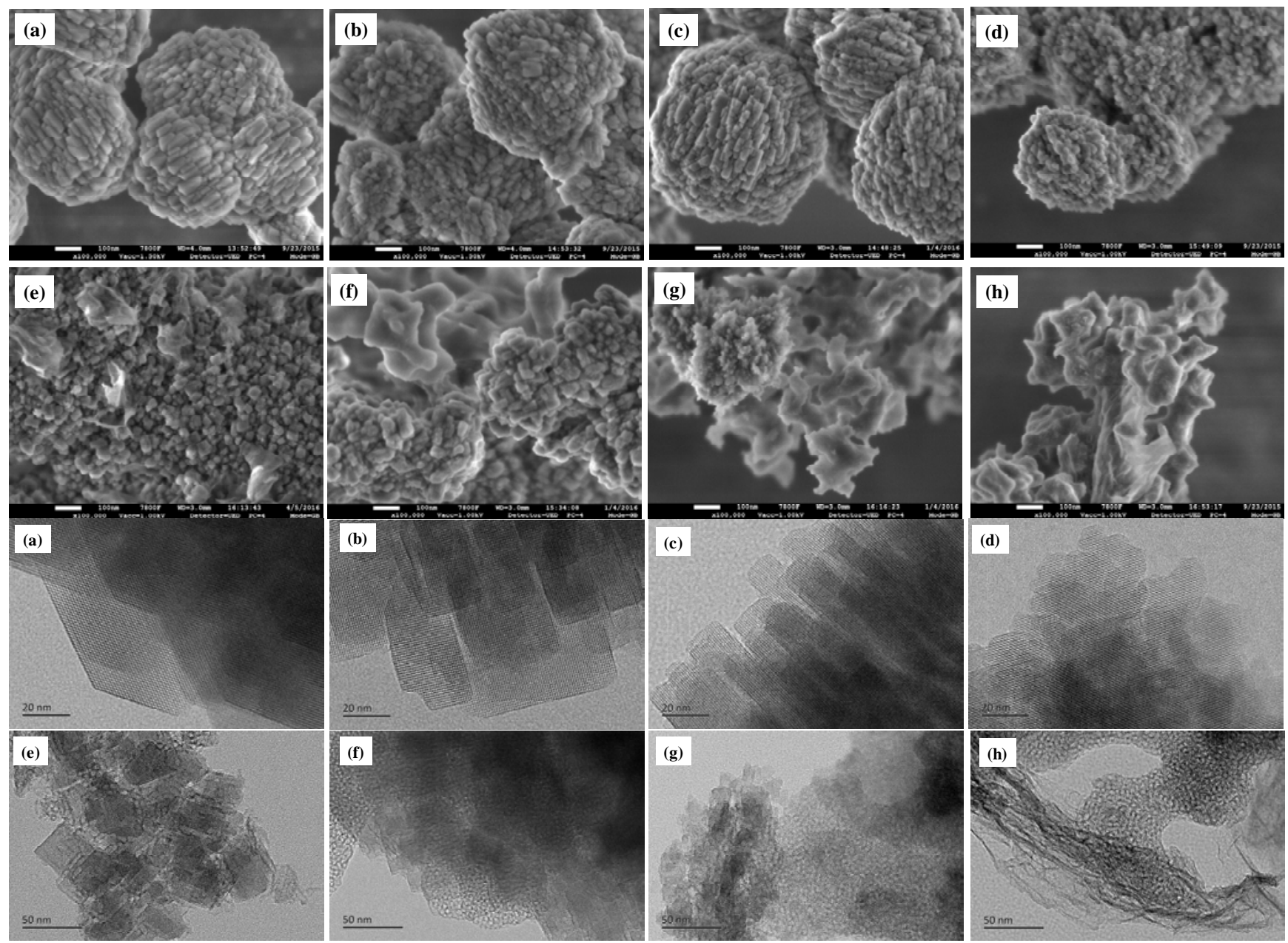

Fig. 2. SEM and TEM images of (a) NaZSM-11, (b) NaZ-CTAB-0.5, (c) NaZ-CTAB-1, (d) NaZ-CTAB-3, (e) NaZ-CTAB-4, (f) NaZ-CTAB-5, (g) NaZ-CTAB-5.2, and (h) NaZ-CTAB-6.

The TEM images of the products (Fig. 2) are consistent with the stepwise structural changes discussed above. The NaZSM-11 zeolite showed a clear crystal lattice and neat crystal edges, indicating high crystallinity. The crystal sizes of the samples synthesized with the addition of CTAB, i.e., the NaZ-CTAB- $R$ samples, decreased with increasing $R$, and the crystals had curved edges. When $R$ reached 3 , the crystal grains became irregular. In accordance with the SEM image, the TEM image of NaZ-CTAB-4 showed a small amount of a poorly ordered hexagonal mesostructured material, and the zeolite phase predominated in this case. When $R$ was further increased, the proportion of the mesoporous phase increased, and Z-CTAB- 6 contained only a mesoporous phase. This hexagonal mesoporous phase was also observed in a synthesized hierarchical MWW zeolite [42]. In the case of a MOR zeolite synthesized in the presence of CTAB and poly(ethylene glycol) (PEG), a hierarchical MOR with a nanosheet structure was the sole product, without an amorphous phase, because PEG surrounded the exterior of the CTAB micelles, which restricted the self-assembly of [ $\left.\mathrm{SiO}_{4}\right]$ into an amorphous material [43]. The TEM and SEM images (Fig. 2) are clearly consistent with the XRD results (Fig. 1). It was worth noting that mesoporous MCM-41 samples synthesized under different conditions had different morphologies [44-46]. The SEM images showing an MCM-41-like phase in NaZ-CTAB- $R$ are therefore reasonable (Fig. 2). The poorly ordered mesoporosity, verified by the TEM images (Fig. 2), was also different from those reported previously $[45,47,48]$. The TEM images (Fig. 2) clearly show that none of the NaZ-CTAB- $R$ samples contained intracrystalline mesopores.

\subsection{Textural and physicochemical characterization}

The addition of a mesoporous template to the reaction gel not only alters the morphology but also significantly affects the textural and physicochemical properties of the final products. The data in Table 1 show that the $\mathrm{Si} / \mathrm{Al}_{2}$ molar ratio of the resultant samples depended on the $R$ value. The $\mathrm{Si} / \mathrm{Al}_{2}$ molar ratio clearly increased with increasing $R$, for $R<3$. When $R$ was further increased from 4 to $5.2, \mathrm{Si} / \mathrm{Al}_{2}$ remained nearly constant. An $R$ of 6 gave a higher $\mathrm{Si} / \mathrm{Al}_{2}$. However, inductively coupled plasma optical emission spectroscopy indicated that the overall $\mathrm{Si} / \mathrm{Al}_{2}$ ratios for the hierarchical ZSM-5 zeolite samples with different $n$ (TPAOH) $/ n$ (CTAB) values were nearly the same [49]. The trend in the changes in $\mathrm{Si} / \mathrm{Al}_{2}$ was almost the opposite of the trend in the RC. Furthermore, $\mathrm{Si} / \mathrm{Al}_{2}$ showed an interest- 
ing relationship with the structural and morphological changes. For Z-CTAB- $R(R=0.25-3), \mathrm{Si} / \mathrm{Al}_{2}$ increased with decreasing length of the rod-like nanoparticles and increasing mesoporosity. For Z-CTAB- $R(R=4-5.2), \mathrm{Si} / \mathrm{Al}_{2}$ remained nearly constant; the products contained a combination of microporous and mesoporous phases, and their mesoporosities did not obviously change. For Z-CTAB-6, both $\mathrm{Si} / \mathrm{Al}_{2}$ and the mesoporosity clearly increased, and the sample consisted mainly of a poorly ordered hexagonal mesoporous phase.

Fig. 3 shows the $\mathrm{N}_{2}$ adsorption and desorption isotherms and the Barrett-Joyner-Halenda (BJH) pore size distribution (PSD) curves derived from the adsorption branch of the HZSM-11 and HZ-CTAB-R samples. HZSM-11 gave a typical type I isotherm at a low relative pressure, which is characteristic of a microporous zeolite (Fig. 3(a)). The small amount of mesopores in HZSM-11, shown in its BJH PSD plot (Fig. 3(b)), could be derived from intercrystalline mesopores [7]. At low levels of CTAB addition $(R<3)$, HZ-CTAB- $R$ gave a combination of type I and type IV isotherms, with micropore filling at low pressures and hysteresis loops at $P / P_{0}$ higher than 0.5 , indicating formation of a hierarchical porous system combining microporosity and mesoporosity. These hysteresis loops are usually associated with capillary filling and condensation of $\mathrm{N}_{2}$ within the homogeneous slit-shaped intercrystalline meso- pores formed by aggregation of nanosized zeolite crystals [40]. The BJH PSD plots show a bimodal pore distribution, with a small pore diameter, i.e., less than $10 \mathrm{~nm}$, and a larger pore diameter at 10-20 $\mathrm{nm}$. The band representing the smaller mesopore shifted from ca. $4 \mathrm{~nm}$ to ca. $8 \mathrm{~nm}$ and the intensity of both bands increased with increasing $R$ from 0.25 to 3 . Addition of more CTAB $(R=4-4.3)$ led to a hysteresis loop at $P / P_{0}$ higher than 0.4 for the HZ-CTAB- $R$ samples and their BJH PSD plots showed a weaker double pore distribution, with the smaller pore diameter shifting to ca. 3-4 $\mathrm{nm}$ and the larger one increasing to ca. $25 \mathrm{~nm}$. Fig. 3(c) shows that when $R$ was higher than 4.5 , the HZ-CTAB- $R$ isotherm increased sharply at $P / P_{0}=$ $0.3-0.45$, indicating the presence of uniform mesopores of diameter 3-4 $\mathrm{nm}[50,51]$ or framework-confined mesopores [52]; this was verified by the BJH PSD plot (Fig. 3(d)) and the small-angle XRD pattern, which showed a broad and intense peak at $2 \theta=2^{\circ}$. In addition, with increasing $R$ from 4.5 to 6 , only one clear band, at ca. 3-4 nm, was present in the BJH PSD curves, and the intensity increased significantly with increasing $R$.

The textural property data for all the samples are shown in Table 1. Compared with that of HZSM-11, with only micropores, the introduction of mesoporosity by CTAB addition led to a progressive decrease in the microporous volume $\left(V_{\mathrm{mi}}\right.$
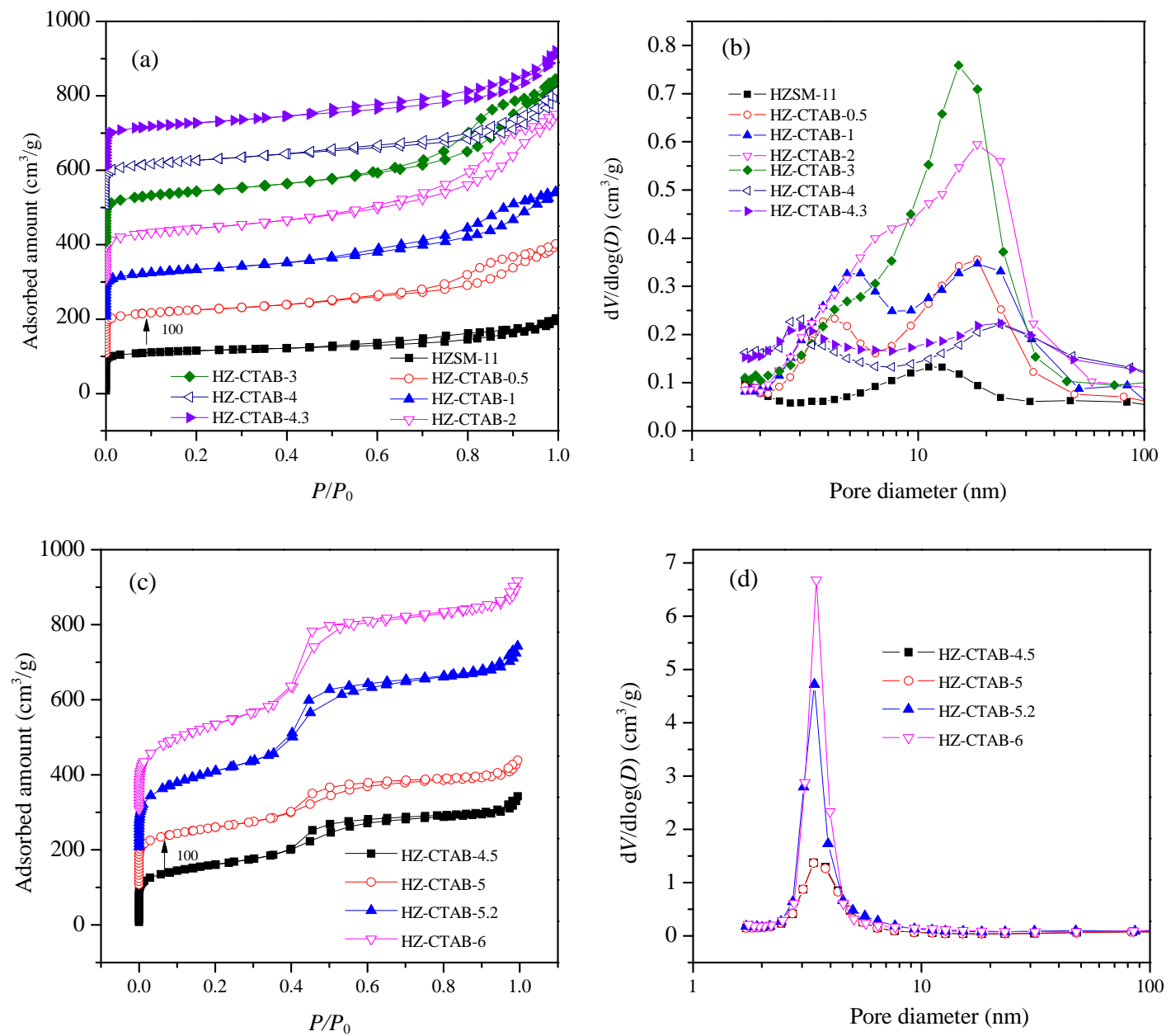

Fig. 3. $\mathrm{N}_{2}$ adsorption and desorption isotherms $(\mathrm{a}, \mathrm{c})$ and $\mathrm{BJH}$ pore size distribution plots (b, d) derived from adsorption branch for various samples. 
cro) from 0.132 to $0.016 \mathrm{~cm}^{3} / \mathrm{g}$ with increasing $R$ from 0.25 to 6 . When $R$ was less than 3 , the mesoporous volume $\left(V_{\text {meso }}\right)$ of HZ-CTAB- $R$ increased with increasing $R$, and HZ-CTAB-3 had a $V_{\text {meso }}$ of $0.577 \mathrm{~cm}^{3} / \mathrm{g}$. However, the $V_{\text {meso }}$ values of HZ-CTAB-4 and HZ-CTAB-4.3 were 0.378 and $0.394 \mathrm{~cm}^{3} / \mathrm{g}$, respectively, significantly lower than that of HZ-CTAB-3. Further increasing $R$ from 4 to 6 resulted in a continuous increase in $V_{\text {meso }}$ of HZ-CTAB- $R$. In SAPO-34 synthesis, CTAB addition decreased the microporosity and increased the mesoporosity [39]. The BET surface areas $\left(A_{\mathrm{BET}}\right)$ of HZ-CTAB- $R$ increased with increasing $R$, except in the cases of HZ-CTAB- 4 and HZ-CTAB-4.3. The morphologies and structures discussed above suggest that the decreased surface areas of HZ-CTAB- 4 and HZ-CTAB-4.3 were caused by close packing of the microporous and mesoporous phases. The significant decrease in $V_{\text {meso }}$ for both the samples support this speculation.

In the design of hierarchical zeolites, enhancing the mesoporous surface area $\left(A_{\text {meso }}\right)$ without severe loss of $V_{\text {micro }}$ is essential. The hierarchical factor (HF), defined as $\left(V_{\text {micro }} / V_{\text {total }}\right) \times$ ( $A_{\text {meso }} / A_{\mathrm{BET}}$ ), which is one of the most important factors in describing hierarchical zeolites [5], was calculated for most of the samples (Table 1). HZSM-11 gave the lowest HF, i.e., 0.011, because of the absence of mesostructures. The HF increased with increasing $R$ from 0.25 to 3 as a result of the introduction of intercrystalline mesoporosity. However, on further increasing $R$, the sample HFs decreased; this could be attributed to a significant loss of microporosity, in spite of the increasing mesoporosity.

Previously, we reported that alkaline treatment of a ZSM-11 zeolite with $\mathrm{NaOH}$ and CTAB produced uniform intracrystalline mesopores, with the PSD centered at ca. $4.2 \mathrm{~nm}$, with good protection of the microporosity [7]. This can be ascribed to the drilling effect of CTAB under alkaline conditions [53]. However, there are significant differences between the results for ZSM-11 synthesis with CTAB and ZSM-11 alkaline treatment with CTAB. Here, ZSM-11 synthesis in the presence of CTAB did not produce intracrystalline mesopores, as verified by the TEM images (Fig. 2). The HZ-CTAB- $R(R=0.5-3)$ contained mesopores centered at ca. $4-8 \mathrm{~nm}$, which could be intercrystalline mesopores, and can probably be attributed to organic CTAB molecules being loosely incorporated between crystal grains [42].

CTAB is well known for its mesopore-directing effect in the synthesis of mesoporous silica [54]. However, CTAB failed to function as a mesopore-directing agent when it was added to zeolite synthesis systems in attempts to obtain mesoporous materials with crystalline zeolite frameworks, as previously reported $[38,55,56]$; the obtained samples were composites of micro- and meso-porous materials. CTAB micelles were therefore expelled from the aluminosilicate domain during the zeolite crystallization process, making it difficult for the surfactant to modulate zeolite crystal growth to give a mesoporous structure [18].

\subsection{Effect of alkalinity on zeolite synthesis}

As discussed in our previous papers, the MCM-41-like phase is sensitive to the alkalinity during post-treatment with a solution of $\mathrm{NaOH}$ and CTAB $[28,29]$. We wondered if this situation also applied one-pot zeolite synthesis. The effect of alkalinity on hierarchical ZSM-11 zeolite synthesis in the presence of CTAB was therefore investigated.

TEM images showed that NaZ-CTAB-5 contained a large MCM-41-like mesoporous phase (Fig. 4). NaZ-CTAB-5a, which was obtained by increasing the alkalinity, contained a significantly smaller mesoporous phase. When the alkalinity was increased further, the MCM-41-like phase in NaZ-CTAB-5b disappeared. In addition, the crystal grains became larger and the borders became neater with increasing alkalinity. These results are in good accordance with those in the literature [28], and show that in alkaline treatment with CTAB, an MCM-41-like phase can be formed by decreasing the $\mathrm{pH}$. These results suggest that higher alkalinity suppresses the formation of an MCM-41-like phase in zeolite synthesis with CTAB or zeolite alkaline treatment with CTAB.

The wide-angle XRD patterns (Fig. 5(a)) show that the crystallinity of the Z-CTAB-5 series samples increased with increasing alkalinity. The small-angle XRD patterns (Fig. 5(b)) show that the intensity of the peak at $2 \theta=2^{\circ}$ decreased with increasing alkalinity; this is ascribed to a decrease in the amount of MCM-41-like phase. In particular, NaZ-CTAB-5b had high crystallinity and almost no peak at $2 \theta=2^{\circ}$. These results are in agreement with the TEM results (Fig. 4), and further verify that a higher alkalinity suppresses formation of the MCM-41-like phase under these circumstances.

The $\mathrm{N}_{2}$ adsorption and desorption isotherms of HZ-CTAB- 5 and HZ-CTAB-5a changed sharply at about $0.3<P / P_{0}<0.45$ (Fig. 6(a)), and their BJH PSD curves showed a mesopore size of 3-4 nm (Fig. 6(b)), indicating the presence of uniform mesopores $[50,51]$ or framework-confined mesopores [52]. Howev-
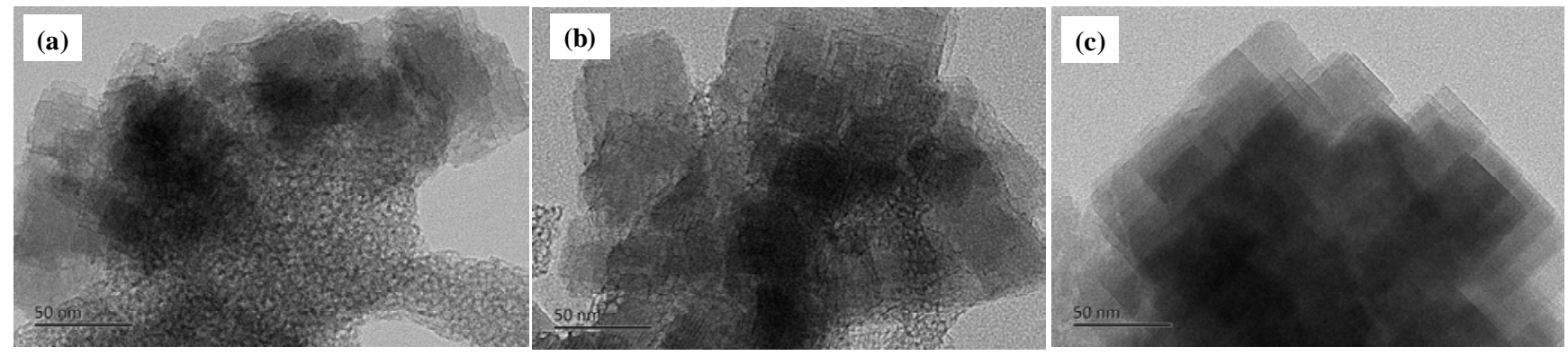

Fig. 4. TEM images of NaZ-CTAB-5 series samples synthesized at various alkalinities. (a) NaZ-CTAB-5; (b) NaZ-CTAB-5a; (c) NaZ-CTAB-5b. 

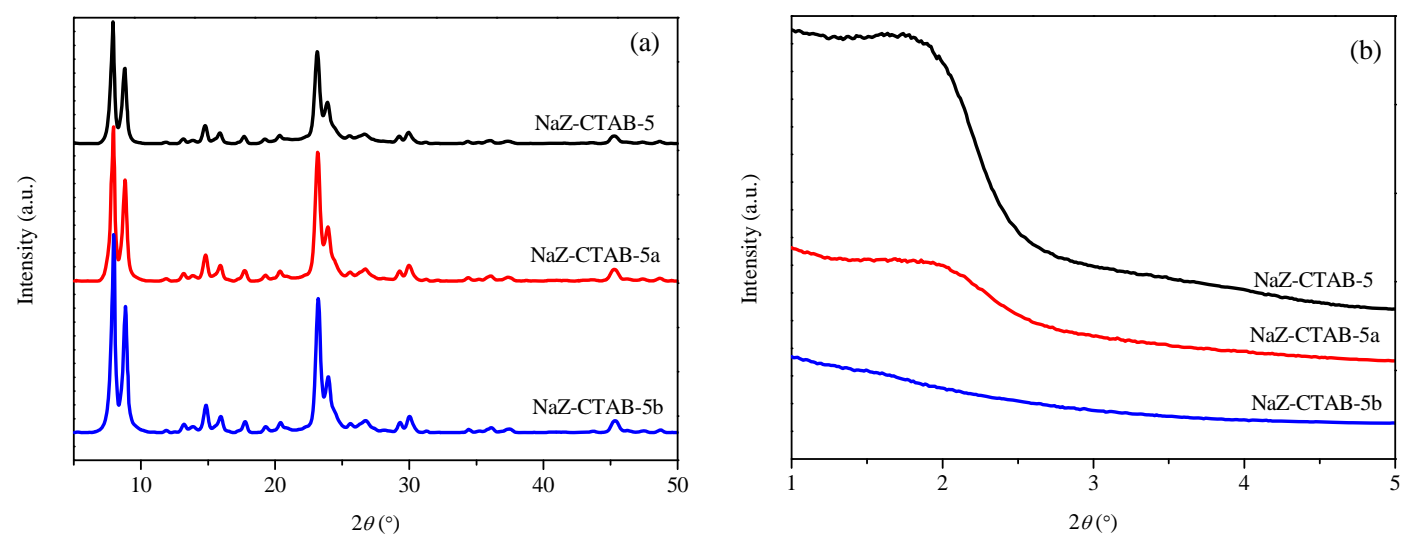

Fig. 5. Wide-angle (a) and small-angle (b) XRD patterns of NaZ-CTAB-5 series samples synthesized at various alkalinities.
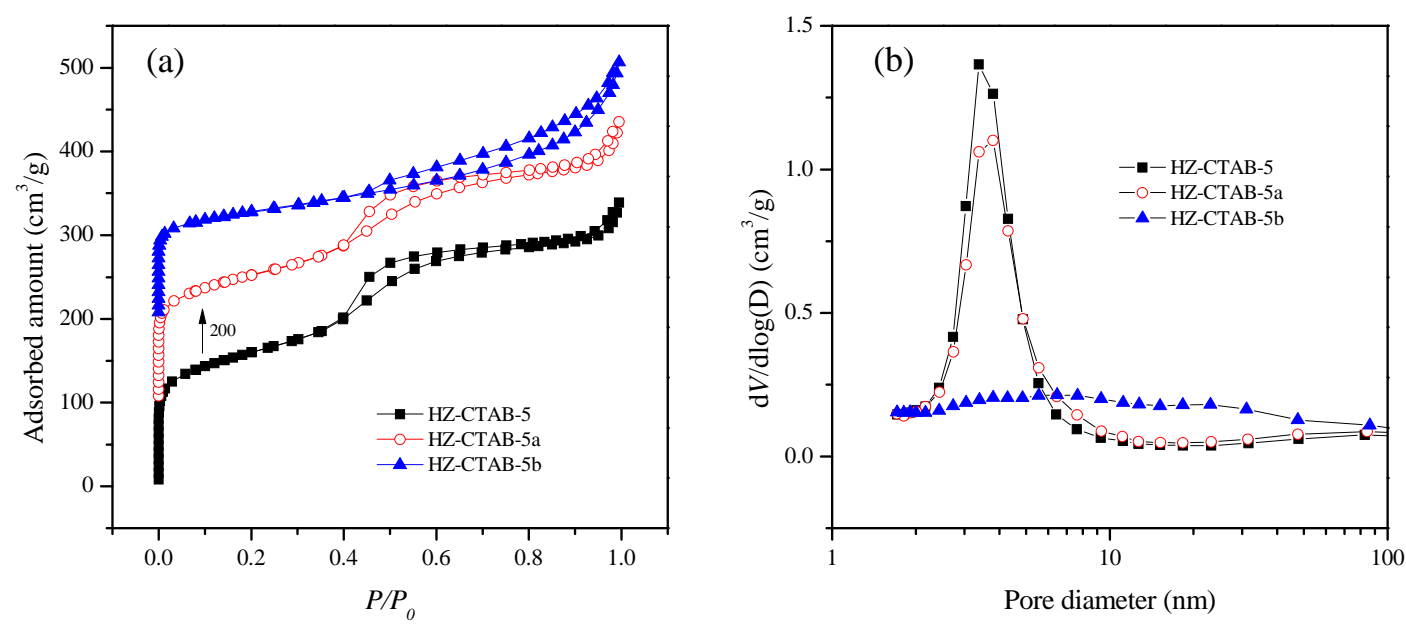

Fig. 6. $\mathrm{N}_{2}$ adsorption and desorption isotherms (a) and BJH pore size distribution plots (b) derived from adsorption branch for $\mathrm{HZ}$-CTAB-5 series samples synthesized at various alkalinities.

er, contrary to reports in the literature [52], the isotherms of HZ-CTAB-5 and HZ-CTAB-5a in the $P / P_{0}$ range $0.3-0.45$ did not show a nearly overlapping hysteresis loop, and the PSD was wider. This may be caused by the presence of poorly ordered hexagonal mesostructured materials. HZ-CTAB- $5 \mathrm{~b}$ gave a hysteresis loop at $P / P_{0}$ higher than 0.45 , which probably arises from intercrystalline mesopores. This can be explained by the BJH PSD curve, which shows a broad and weak band at ca. 4-20 $\mathrm{nm}$. No intracrystalline mesopores can be observed in the TEM image (Fig. 4(c)). These mesopores probably originate from the gaps between spherical aggregated structures or from the micellar effect of CTAB [42]. The textural data indicate that the $V_{\text {micro }}$ and $V_{\text {meso }}$ values for the HZ-CTAB-5 series samples increased and decreased, respectively, with increasing alkalinity (Table 1).

The results show that the alkalinity of the reaction mixture significantly affected hierarchical ZSM-11 zeolite synthesis. With increasing alkalinity, the structure-directing effect of TBABr was enhanced and the effect of CTAB was weakened, resulting in increased ZSM-11 crystallinity and a decreased amount of the MCM-41-like phase. A relatively high alkalinity was beneficial in obtaining ZSM-11 crystals with good regularity. This shows that the effect of increasing the alkalinity of the reaction mixture was similar to that of decreasing the $R$ value, described above. Zeolites such as ZSM-5 and ZSM-11 have usually been obtained at high $\mathrm{pH}$ values $[1,25]$. At lower $\mathrm{pH}$ values, the ZSM-5 zeolite was not be obtained in the presence of CTAB, and the product was ordered MCM-41; with increasing $\mathrm{pH}$, ZSM-5 was gradually formed in the products [25]. This is consistent with our work.

\subsection{Formation mechanism of hierarchical ZSM-11 zeolite}

A possible mechanism for the formation of hierarchical ZSM-11 zeolites is proposed on the basis of the characterization results (Scheme 1). The following simplifications were made to better illustrate the effects of $\mathrm{TBABr}$ and $\mathrm{CTAB}$ during zeolite synthesis. ZSM-11 zeolite was formed under the direction of the microporogen $\mathrm{TBA}^{+}$(i.e., a structure-directing effect) $[9,57]$. CTAB played various roles, depending on the circumstances. The long alkyl tail of CTAB, which is hydrophobic, could link with the surfaces of nanocrystals and prevent the primary nanosized zeolite crystals from growing further (i.e., a capping effect) $[40,58]$. CTAB was sufficient to generate free micelles (i.e., the micellar effect) because the critical micelle concentration was less than $0.001 \mathrm{~mol} / \mathrm{L}$ [59]. Furthermore, CTAB could 


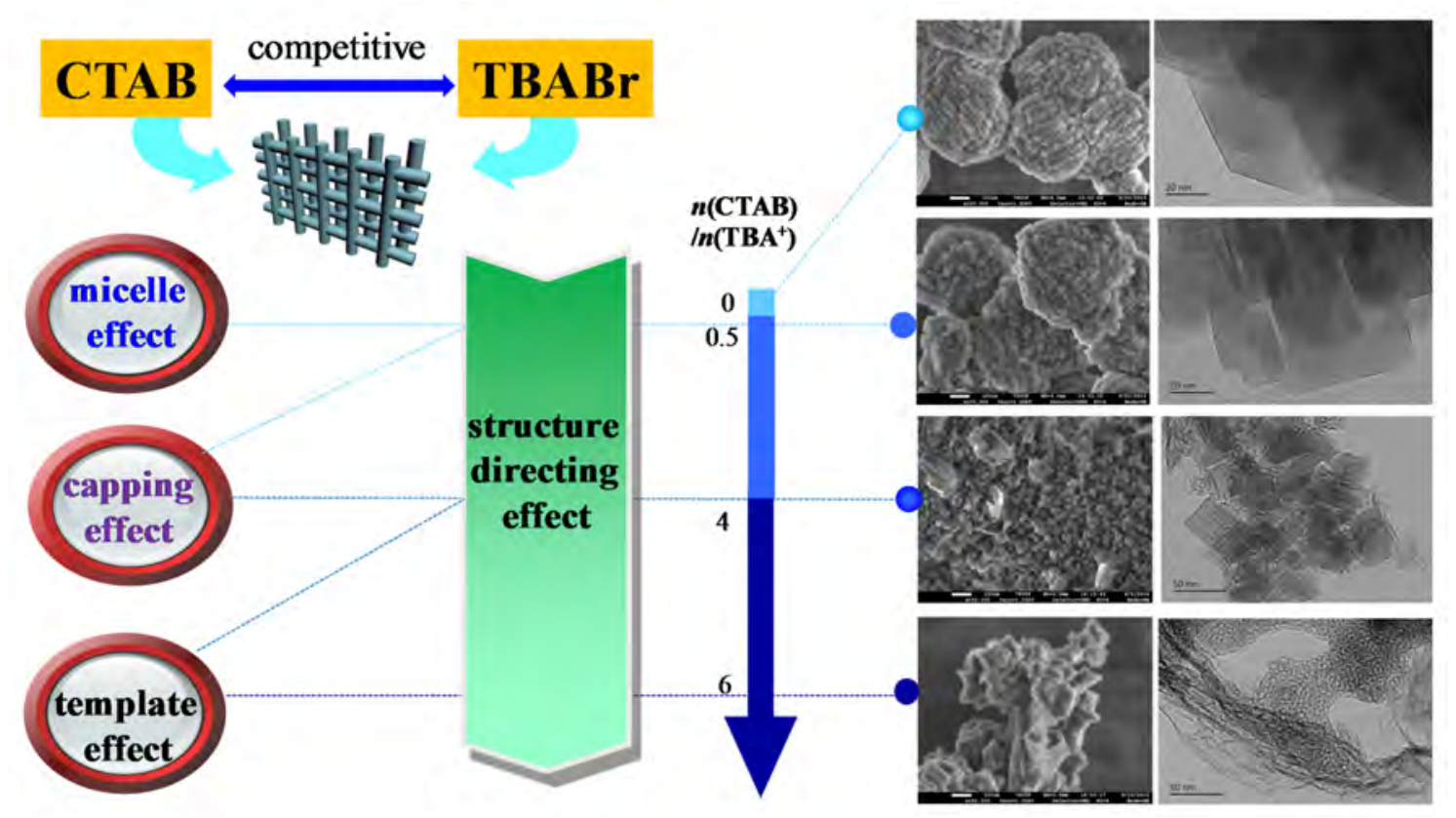

Scheme 1. Formation mechanism of hierarchical ZSM-11 zeolite.

act as a mesopore template when the CTAB concentration was high enough, to form a MCM-41-like phase (i.e., a template effect) $[18,60,61]$.

The ratio of CTAB to TBAB $(R)$ in the reaction mixture was clearly important in formation of the hierarchical zeolite. (i) $\mathrm{TBA}^{+}$gave a structure-directing effect without CTAB addition (HZSM-11). (ii) The capping effect and micellar effect of CTAB were coexistent, whereas these effects competed with the structure-directing effect of $\mathrm{TBA}^{+}$when the amount of added CTAB was low $(R=0.25-3)$. (iii) CTAB showed simultaneous capping and template effects, which inhibited the structure-directing effect of $\mathrm{TBA}^{+}$when a larger amount of CTAB was added $(R=4-5.2)$. (iv) CTAB gave a template effect only when its concentration increased to a certain value, and completely suppressed the structure-directing effect of $\mathrm{TBA}^{+}(R=$ 6).

As shown in Fig. 2, HZSM-11 showed a clear crystal lattice and neat crystal edges. When the amount of CTAB added was low, HZ-CTAB- $R(R=0.25-3)$ had small crystalline grains with curved crystal edges because of the capping effect. HZ-CTAB- $R$ had a bimodal PSD (Fig. 3). For convenience, the band representing mesopores of size less than $10 \mathrm{~nm}$ is labeled as Band I, and that representing mesopores of size greater than $10 \mathrm{~nm}$ is labeled as Band II. None of the samples contained intracrystalline mesopores (Fig. 2), therefore Bands I and II were probably both derived from intercrystalline mesopores. Specifically, Band I could be the result of crystalline grains assembling around CTAB micelles; the mesopore size increase from 4 to 8 $\mathrm{nm}$ with increasing $R$ from 0.25 to 3 , probably because of micelle agglomeration. Band II could be related to gaps in the spherical aggregated structure, because the HZSM-11 zeolite with only mesoporosity showed a weak band from intercrystalline mesopores at 10-20 nm. For HZ-CTAB- $R(R=0.25-3)$, the position of Band II was almost constant, and its intensity in- creased with increasing $R$.

It can therefore be deduced that during zeolite synthesis with CTAB addition $(R=0.25-3)$, the capping and micellar effects of CTAB coexisted. Moreover, CTAB addition gave rise to a continuous decrease in RC and $V_{\text {micro, which can be attributed to }}$ attenuation of the structure-directing effect of $\mathrm{TBA}^{+}$. The effects of CTAB and $\mathrm{TBA}^{+}$during ZSM-11 zeolite synthesis were therefore competitive. Similarly, in the case of ZSM-5 zeolite synthesis, microporogens and mesoporogens worked in a competitive, rather than a cooperative, manner $[22,56,60,61]$. The electrostatic force between the positively charged CTA+ micelle and the negatively charged zeolite crystal is weak, therefore seed crystals cannot effectively assemble around $\mathrm{CTA}^{+}$micelles, which hinders zeolite crystallization, leading to reductions in the RCs and microporosities of the zeolite samples [61]. CTAB also retards nucleation and crystallization of the MFI structure, leading to production of an amorphous phase [38].

On further increasing $R$ to 4, an MCM-41-like phase appeared, as shown by the SEM and TEM images in Fig. 2. A weak peak at ca. $2 \theta=2^{\circ}$ appeared in the small-angle XRD pattern of NaZ-CTAB-4, despite the absence of a clear peak in Fig. 1, because of a small amount of the MCM-41-like phase. The BJH PSD curve of HZ-CTAB-4 showed a pore diameter of ca. 3-4 nm, i.e., smaller than that of HZ-CTAB-3 (Fig. 3(b)). These MCM-41-like phases could induce the template effect of CTAB; similar results have been reported in a review [60]. The zeolite phase was the main phase in HZ-CTAB-4. The capping effect of CTAB was still present, because the crystal grains of HZ-CTAB-4 were clearly smaller than those of HZSM-11. However, the micellar effect of CTAB was weakened. This is shown by the BJH PSD curve of HZ-CTAB-4; band I shows a pore diameter of ca. 3-4 nm, attributed to a MCM-41-like phase, and no clear band from the micellar effect of CTAB is observed at ca. $4-8 \mathrm{~nm}$, i.e., the capping effect and template effect of CTAB coexisted, but its 
micellar effect was weakened in this case. The RC and proportion of the zeolite phase in HZ-CTAB- $R(R=4-5.2)$ continued to decrease with increasing $R$ (Table 1 and Fig. 1) because the structure-directing effect of TBA+ was further weakened.

The SEM and TEM images (Fig. 2) verify that there was only a poorly ordered mesoporous phase in the HZ-CTAB-6 sample, because of the template effect of CTAB. The capping effect and micellar effect of CTAB were absent, and the structure-directing effect of $\mathrm{TBA}^{+}$was completely suppressed by excess CTAB. In this case, the ZSM-11 zeolite was almost undetected using XRD (Fig. 1), and the BJH PSD curve for HZ-CTAB-6 showed only a sharp band centered at ca. 3-4 $\mathrm{nm}$ (Fig. 3).

In the case of ZSM-5 synthesis in the presence of CTAB, either a mesoporous phase or large zeolites, or a mixture of the two, is formed, depending on the experimental parameters $[22,56]$. According to a review [37], first, spherical micelles are formed at a low $R$ value and fill the gaps in the ZSM-11 crystal grains to produce intercrystalline mesopores, and then cylindrical micelles form a hexagonal array of parallel rods, which results in formation of an MCM-41-like material. Because of the concentration limitation, no cubic MCM-48 and lamellar MCM-50 mesophases were observed in this work.

\subsection{Acidity characterization}

$\mathrm{NH}_{3}$ temperature-programmed desorption ( $\left.\mathrm{NH}_{3}-\mathrm{TPD}\right)$ and Py-IR spectroscopy were used to investigate the acidic properties of the synthesized samples. The total acidities and distributions of acid sites with different strengths are listed in Table 2. Fig. 7 shows that the HZSM-11 and hierarchical HZ-CTAB- $R(R$ $=0.25-5$ ) samples all gave two well-resolved desorption peaks (a low-temperature peak at ca. $250{ }^{\circ} \mathrm{C}$ and a high-temperature peak at ca. $450{ }^{\circ} \mathrm{C}$, corresponding to weak acid sites and strong acid sites, respectively). HZ-CTAB-5.2 and HZ-CTAB- 6 gave only one clear peak, representing weak acid sites. The high $\mathrm{Si} / \mathrm{Al}_{2}$ ratios in Z-CTAB-5.2 and Z-CTAB-6, and the tendency of CTAB to complex with Si species to form silicate-CTAB species [59], suggest that the weak acidity was caused by the formation of an amorphous mesoporous phase that lacked lattice $\mathrm{Al}$ sites [16]. The total acid concentration for HZSM-11 was $396 \mu \mathrm{mol} / \mathrm{g}$. For the HZ-CTAB- $R$ samples, the total acid concentration decreased from 435 to $138 \mu \mathrm{mol} / \mathrm{g}$ with increasing $R$ from 0.25 to 6 . The total acid concentration for all the HZ-CTAB-R samples, except

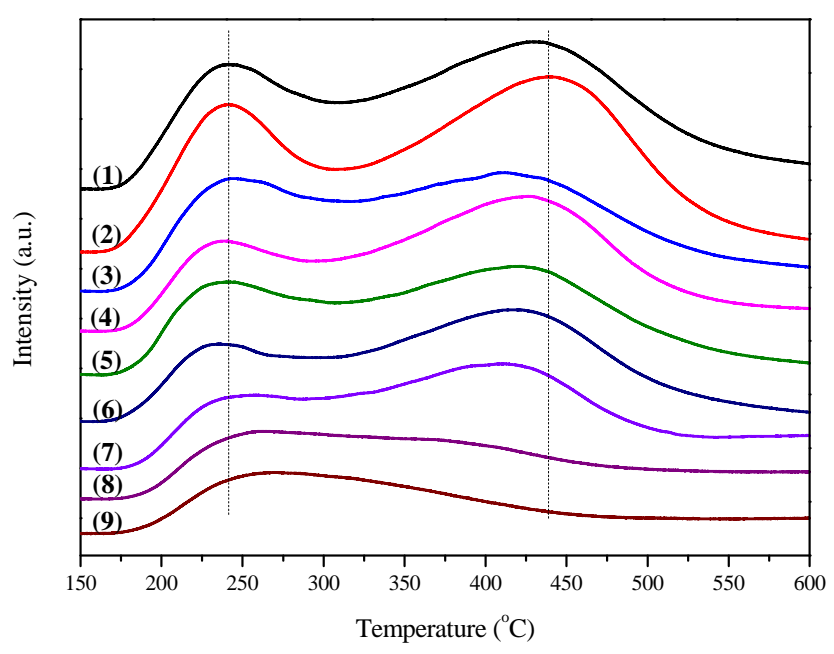

Fig. 7. $\mathrm{NH}_{3}$-TPD curves for (1) HZSM-11, (2) HZ-CTAB-0.25, (3) HZ-CTAB-0.5, (4) HZ-CTAB-1, (5) HZ-CTAB-3, (6) HZ-CTAB-4, (7) HZ-CTAB-5, (8) HZ-CTAB-5.2, and (9) HZ-CTAB-6.

HZ-CTAB-0.25, was lower than that of HZSM-11. A possible reason is that the surfactant molecules inhibited Al incorporation into the zeolite framework to some extent $[23,40]$. The higher acid concentration for HZ-CTAB-0.25 can be ascribed to the low CTAB concentration, which could slightly inhibit formation of a crystalline zeolite phase by silica and alumina species, and lead to formation of a small amount of amorphous silica-alumina (ASA) outside the zeolite; ASA would also contain acid sites [62].

Py-IR spectroscopy was used for further investigation of the distributions of different types of acid sites in the HZSM-11 and HZ-CTAB- $R$ samples; the results are summarized in Table 2. The trends in the changes in the concentrations of $\mathrm{B}+\mathrm{L}$ acids of different strengths, i.e., total (desorbed at $150{ }^{\circ} \mathrm{C}$ ), medium (desorbed at $300{ }^{\circ} \mathrm{C}$ ), and strong acid sites (desorbed at 450 ${ }^{\circ} \mathrm{C}$ ), denoted by $C_{\text {Total }}-150, C_{\text {Total-300, }}$ and $C_{\text {Total-450, }}$ respectively, were similar to those for the total acid concentrations obtained by $\mathrm{NH}_{3}$-TPD. Specifically, the changes in the total, medium, and

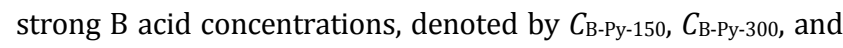

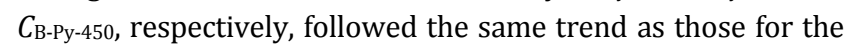
$\mathrm{B}+\mathrm{L}$ acid concentrations. In contrast, the total, medium, and

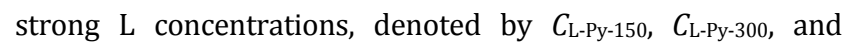
$C_{\mathrm{L}-\mathrm{Py}-450,}$, respectively, first decreased and then increased slight-

Table 2

Acidic properties of the samples determined using $\mathrm{NH}_{3}$-TPD and Py-IR spectroscopy.

\begin{tabular}{|c|c|c|c|c|c|c|c|c|c|c|c|c|c|}
\hline \multirow{2}{*}{ Sample } & \multirow{2}{*}{$\begin{array}{c}\text { Concentration of } \\
\text { acid sites }{ }^{a}(\mu \mathrm{mol} / \mathrm{g})\end{array}$} & \multicolumn{12}{|c|}{ Concentration $(\mu \mathrm{mol} / \mathrm{g})$ and ratio of acid sites ${ }^{b}$} \\
\hline & & $C_{\mathrm{B}-\mathrm{-Py}-150}$ & $C_{\mathrm{L}-\mathrm{Py}-150}$ & $C_{\text {Total-150 }}$ & $\mathrm{B} / \mathrm{L}_{-150}$ & $C_{\mathrm{B}-\mathrm{Py}-300}$ & $C_{\mathrm{L}-\mathrm{Py}-300}$ & $C_{\text {Total-300 }}$ & B/L-300 & $C_{\mathrm{B}-\mathrm{Py}-450}$ & $C_{\mathrm{L}-\mathrm{Py}-450}$ & $C_{\text {Total-450 }}$ & B/L-450 \\
\hline HZSM-11 & 396 & 96 & 81 & 177 & 1.19 & 91 & 41 & 132 & 2.22 & 58 & 32 & 90 & 1.81 \\
\hline HZ-CTAB- 0.25 & 435 & 118 & 88 & 206 & 1.34 & 107 & 46 & 153 & 2.33 & 75 & 41 & 116 & 1.83 \\
\hline HZ-CTAB-0.5 & 345 & 81 & 71 & 152 & 1.14 & 70 & 37 & 107 & 1.89 & 51 & 29 & 80 & 1.76 \\
\hline HZ-CTAB-1 & 327 & 74 & 69 & 143 & 1.07 & 61 & 35 & 96 & 1.74 & 40 & 26 & 66 & 1.52 \\
\hline HZ-CTAB-3 & 289 & 64 & 62 & 126 & 1.03 & 58 & 34 & 92 & 1.71 & 36 & 23 & 59 & 1.57 \\
\hline HZ-CTAB-4 & 277 & 56 & 55 & 111 & 1.02 & 44 & 26 & 70 & 1.69 & 35 & 22 & 57 & 1.59 \\
\hline HZ-CTAB-5 & 273 & 52 & 58 & 110 & 0.90 & 43 & 33 & 76 & 1.30 & 32 & 30 & 62 & 1.07 \\
\hline HZ-CTAB-5.2 & 192 & 39 & 60 & 99 & 0.65 & 22 & 34 & 56 & 0.65 & 12 & 30 & 42 & 0.40 \\
\hline HZ-CTAB-6 & 138 & 32 & 66 & 98 & 0.48 & 19 & 36 & 55 & 0.53 & 4 & 31 & 35 & 0.13 \\
\hline
\end{tabular}

${ }^{a}$ Detected using $\mathrm{NH}_{3}$-TPD. ${ }^{\mathrm{b}}$ Detected by Py-IR technique. 
ly with increasing $R$; HZ-CTAB-4 gave the lowest value.

The $\mathrm{B} / \mathrm{L}$ ratios all decreased with increasing $R$, indicating that ZSM-11 synthesis in the presence of CTAB led to a higher loss of $B$ acid sites than of $L$ acid sites. This may be because the presence of CTAB inhibited $\mathrm{Al}$ incorporation into the zeolite framework $[23,40]$. The combined data in Tables 1 and 2 show that destruction of microporous structures in the ZSM-11 framework led to decreased B acidity, but the L acidity did not increase, as previously reported [63-65]. The trends in the B/L values of the samples obtained by the bottom-up method differed from those for the samples obtained by the top-down method [7].

\subsection{Catalytic performance}

The catalytic activities of microporous HZSM-11 and the hierarchical HZ-CTAB- $R$ samples in benzene alkylation with DME were evaluated in a continuous-flow fixed-bed reactor at atmospheric pressure, $350{ }^{\circ} \mathrm{C}, n$ (benzene) $/ n(\mathrm{DME})=2$, and a DME WHSV of $16 \mathrm{~h}^{-1}$. The experimental results show that the product selectivities of the catalysts were similar, in agreement with our previous work [7]. For example, with HZ-CTAB-1 as the catalyst, at a time-on-stream (TOS) of $3 \mathrm{~h}$, the selectivities for dry gases $(<2 \%)$ and liquefied petroleum gas $(<3 \%)$ were low, and the selectivities for liquid products were as high as ca. $95 \%$. Toluene and xylene were the main liquid products, with selectivities of $45.5 \%$ and $32.5 \%$, respectively.

The DME and benzene conversions at TOS values of 3 and $30 \mathrm{~h}$ over various ZSM-11 catalysts are shown in Table 1 . The DME conversion ( $C_{\mathrm{DME}}$ ) at a TOS of $3 \mathrm{~h}$ for all the tested samples were around $97 \%$ to $99 \%$, except in the case of HZ-CTAB-6, which had an amorphous structure and extremely low acidity (Table 2). The benzene conversion $\left(C_{\mathrm{Bz}}\right)$ at a TOS of $3 \mathrm{~h}$ varied depending on the $R$ value. Acidity, especially weak acidity, is a major factor in $C_{\mathrm{Bz}}$ [7]. HZ-CTAB-0.25 had a high strong acid concentration, therefore it gave a low $C_{\mathrm{Bz}}$ compared with that achieved with microporous HZSM-11 (28.50\% vs 38.66\%). Samples with appropriate acidities (concentration and strength), i.e., HZ-CTAB- $R(R=1-4)$, maintained high $C_{\mathrm{Bz}}$ values. However, the weak acidity of HZ-CTAB-6 led to a sharp decline in $C_{\mathrm{Bz}}$, even to negative values. In the benzene-DME reaction system, benzene alkylation (R1) and DME self-aromatization (R2) both occur [35]. The negative benzene conversion over HZ-CTAB- 6 can be attributed to R2 being favored over R1 because self-aromatization of DME can proceed even on a low-acidity zeolite catalyst.

For convenience, the changes in $C_{\mathrm{DME}}$ (or $C_{\mathrm{Bz}}$ ) at TOS values of 3 and $30 \mathrm{~h}$ were used to represent the stabilities of the tested catalysts: the smaller the variation, the higher the stability. Fig. 8 shows that the changes in $C_{\mathrm{DME}}$ and $C_{\mathrm{Bz}}$ followed similar trends when $R$ was less than 4 , but different results were obtained when $R$ was greater than 4 . The stability of a zeolite catalyst is closely related to its porosity and acidity [8]. Specifically, the catalyst stability increased with increasing $R$ from 0 to 0.5 , probably because the improved mesoporosity facilitated coke diffusion. The stability then remained relatively constant with increasing $R$ from 0.5 to 3 . It is worth noting that the

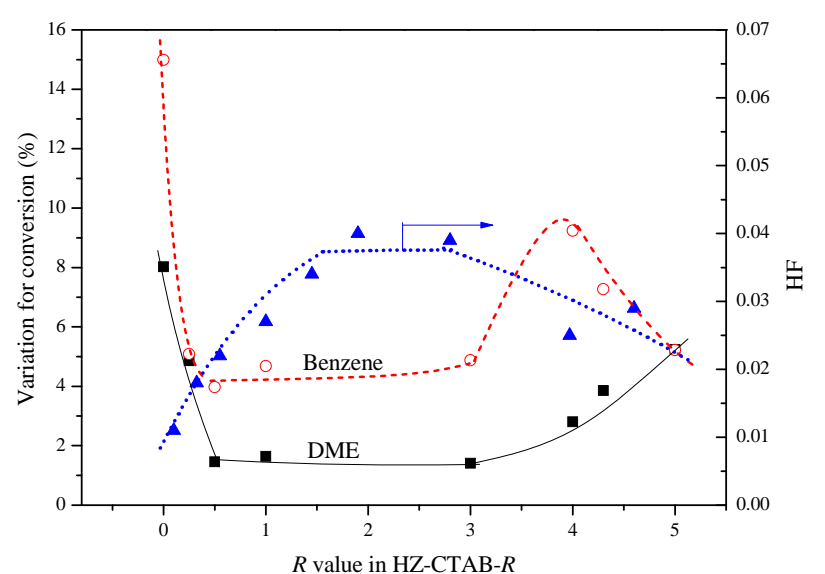

Fig. 8. Changes in DME and benzene conversions and HF as function of $R$ for HZ-CTAB- $R$ samples.

mesoporosities of these samples increased with decreasing acid concentration, irrespective of the acid strength. The constant stability may be the result of a combination of the mesoporosity and acidity. When $R$ was greater than 3 , the catalyst stability decreased in the case of $C_{\mathrm{DME}}$ because of the reduced acidity, but the mesoporosity first decreased and then increased. However, in the case of $C_{\mathrm{Bz}}$, the stability first decreased and then increased; this seems to be more closely associated with the acidity. These results indicate that the stability of the zeolite catalyst was affected by the mesoporosity and the acidity, but these played different roles in different cases. Because of its weak acidity, HZ-CTAB- 6 gave low $C_{\mathrm{DME}}$ and $C_{\mathrm{Bz}}$ values, and its stability was not taken into account. Because of the importance of the HF, we investigated its relationship with the Z-CTAB-R stability (Fig. 8). The HF first increased, then remained constant, and finally decreased with increasing $R$. This trend is similar to that for the catalyst stability in the case of DME. Although there are some differences for $R$ between 0.5 and 2, this result can be ascribed to the joint effects of acidity and porosity, as described above.

\section{Conclusions}

In summary, CTAB and TBABr were used as meso- and micro-templates, respectively, to synthesize a series of hierarchical ZSM-11 zeolite materials with different mesoporosities and acidities via an ordinary one-pot hydrothermal approach, with different CTAB/TPBAr molar ratios $(R)$. The samples changed from a pure microporous ZSM-11 zeolite, to a ZSM-11/MCM-41-like composite material, and finally to an almost pure MCM-41 phase, with increasing $R$. The alkalinity was verified to be an important factor during the synthesis. A mechanism for formation of the hierarchical ZSM-11 was proposed. The various effects of the mesoporogen, i.e., CTAB (capping effect, micellar effect, and template effect) were synergetic, but they competed with the structure-directing effect of the microporogen TBABr. This led to the formation of samples with various structures, morphologies, and acidities, and affected their catalytic performances in benzene alkylation with DME. 


\section{Graphical Abstract}

Chin. J. Catal., 2018, 39: 167-180 doi: 10.1016/S1872-2067(17)62984-X

Synthesis, characterization, and catalytic performance of hierarchical ZSM-11 zeolite synthesized via dual-template route

Hui Liu*, Shuang Zhang, Sujuan Xie, Wanshuo Zhang, Wenjie Xin, Shenglin Liu, Longya Xu*

Dalian Institute of Chemical Physics, Chinese Academy of Sciences;

Sinochem Quanzhou Petrochemical Co., Ltd;

Harbin Normal University

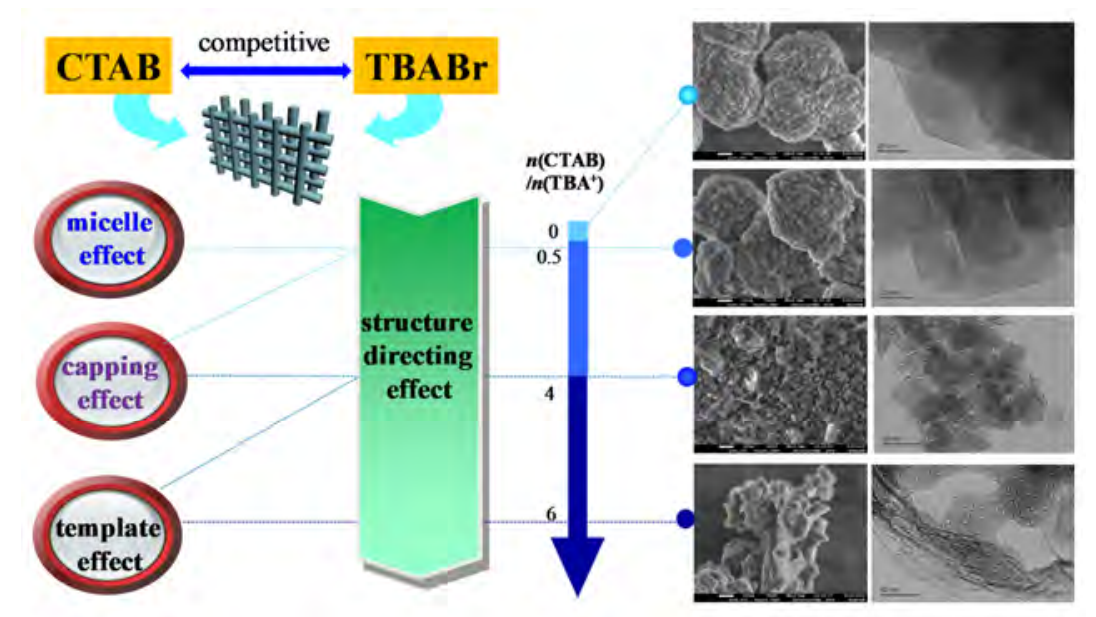

The porosity, structure, morphology, and textural properties of a hierarchical ZSM-11 zeolite were tailored by changing the CTAB/TBABr molar ratio. A mechanism for formation of the hierarchical materials was proposed.

\section{References}

[1] L. Zhang, H. J. Liu, X. J. Li, S. J. Xie, Y. Z. Wang, W. J. Xin, S. L. Liu, L. Y. Xu, Fuel Process. Technol., 2010, 91, 449-455.

[2] W. F. Chu, F. C. Chen, C. Guo, X. J. Li, X. X. Zhu, Y. Gao, S. J. Xie, S. L. Liu, N. Jiang, L. Y. Xu, Chin. J. Catal., 2017, 38, 1880-1887.

[3] S. Y. Gao, X. X. Zhu, X. J. Li, Y. Z. Wang, Y. Zhang, S. J. Xie, J. An, F. C. Chen, S. L. Liu, L. Y. Xu, Chin. J. Catal., 2017, 38, 106-114.

[4] W. S. Zhang, S. Y. Gao, S. J. Xie, H. Liu, X. X. Zhu, Y. C. Shang, S. L. Liu, L. Y. Xu, Y. Zhang, Chin. J. Catal., 2017, 38, 168-175.

[5] J. Pérez-Ramírez, S. Abelló, A. Bonilla, J.C. Groen, Adv. Funct. Mater., 2009, 19, 164-172.

[6] J. Kim, M. Choi, R. Ryoo, J. Catal., 2010, 269, 219-228.

[7] H. Liu, S. J. Xie, W. J. Xin, S. L. Liu, L. Y. Xu, Catal. Sci. Technol., 2016, 6, 1328-1342.

[8] H. Liu, S. L. Liu, S. J. Xie, C. Song, W. J. Xin, L. Y. Xu, Catal. Lett., 2015, 145, 1972-1983.

[9] M. Y. Kustova, P. Hasselriis, C. H. Christensen, Catal. Lett., 2004, 96, 205-211.

[10] K. F. Liu, S. J. Xie, G. L. Xu, Y. N. Li, S. L. Liu, L. Y. Xu, Appl. Catal. A, 2010, 383, 102-111.

[11] R. Baran, Y. Millot, T. Onfroy, J. M. Krafft, S. Dzwigaj, Microporous Mesoporous Mater., 2012, 163, 122-130.

[12] L. Lakiss, F. Ngoye, C. Canaff, S. Laforge, Y. Pouilloux, Z. X. Qin, M. Tarighi, K. Thomas, V. Valtchev, A. Vicente, L. Pinard, J. P. Gilson, C. Fernandez, J. Catal., 2015, 328, 165-172.

[13] A. A. Rownaghi, F. Rezaei, J. Hedlund, Microporous Mesoporous Mater., 2012, 151, 26-33.

[14] H. S. Cho, R. Ryoo, Microporous Mesoporous Mater., 2012, 151,
107-112.

[15] I. Schmidt, A. Boisen, E. Gustavsson, K. Stähl, S. Pehrson, S. Dahl, A. Carlsson, C. J. H. Jacobsen, Chem. Mater., 2001, 13, 4416-4418.

[16] L. Emdadi, Y. Q. Wu, G. H. Zhu, C. C. Chang, W. Fan, T. Pham, R. F. Lobo, D. X. Liu, Chem. Mater., 2014, 26, 1345-1355.

[17] Y. Q. Wu, L. Emdadi, S. C. Oh, M. Sakbodin, D. X. Liu, J. Catal., 2015, 323, 100-111.

[18] M. Choi, H. S. Cho, R. Srivastava, C. Venkatesan, D. H. Choi, R. Ryoo, Nat. Mater., 2006, 5, 718-723.

[19] L. F. Wang, Z. Zhang, C. Y. Yin, Z. C. Shan, F. S. Xiao, Microporous Mesoporous Mater., 2010, 131, 58-67.

[20] D. H. Park, S. S. Kim, H. Wang, T. J. Pinnavaia, M. C. Papapetrou, A. A. Lappas, K. S. Triantafyllidis, Angew. Chem. Int. Ed., 2009, 48, 7645-7648.

[21] M. Choi, K. Na, J. Kim, Y. Sakamoto, O. Terasaki, R. Ryoo, Nature, 2009, 461, 246-249.

[22] Y. Zhu, Z. L. Hua, J. Zhou, L. J. Wang, J. J. Zhao, Y. Gong, W. Wu, M. L. Ruan, J. L. Shi, Chem. Eur. J., 2011, 17, 14618-14627.

[23] H. Feng, X. Y. Chen, H. H. Shan, J. W. Schwank, Catal. Commun., 2010, 11, 700-704.

[24] F. N. Gu, F. Wei, J. Y. Yang, N. Lin, W. G. Lin, Y. Wang, J. H. Zhu, Chem. Mater., 2010, 22, 2442-2450.

[25] H. Li, H. Z. Wu, J. L. Shi, J. Alloys Compd., 2013, 556, 71-78.

[26] H. L. Jin, M. B. Ansari, E. Y. Jeong, S. E. Park, J. Catal., 2012, 291, 55-62.

[27] H. L. Jin, M. B. Ansari, S. E. Park, Chem. Commun., 2011, 47, 7482-7484.

[28] N. N. Gao, S. J. Xie, S. L. Liu, J. An, X. X. Zhu, L. Y. Hu, H. J. Wei, X. J. Li, L. Y. Xu, Catal. Lett., 2014, 144, 1296-1304.

[29] N. N. Gao, S. J. Xie, S. L, Liu, W. J. Xin, Y. Gao, X. J. Li, H. J. Wei, H. Liu, L. Y. Xu, Microporous Mesoporous Mater., 2015, 212, 1-7. 
[30] G. Z. Li, Z. H. Diao, J. D. Na, L. Wang, Chin. J. Chem. Eng., 2015, 23, 1655-1661.

[31] A. Galadima, O. Muraza, Microporous Mesoporous Mater., 2015, $213,169-180$.

[32] G. C. Laredo, J. Castillo, J. O. Marroquin, F. Hernandez, Appl. Catal. A, 2009, 363, 11-18.

[33] K. Takeishi, Y. Akaike, Appl. Catal. A, 2016, 510, 20-26.

[34] H. Liu, H. J. Wei, W. J. Xin, C. Song, S. J. Xie, Z. N. Liu, S. L. Liu, L. Y. Xu, J. Energy Chem., 2014, 23, 617-624.

[35] H. Liu, H. J. Wei, W. J. Xin, S. L. Liu, S. J. Xie, L. Y. Xu, Acta Petrol. Sin. (Petrol. Process. Sect.), 2014, 30, 115-120.

[36] R. A. García-Muñoz, D. P. Serrano, G. Vicente, M. Linares, D. Vitvarova, J. Čejka, Catal. Today, 2015, 243, 141-152.

[37] C. Perego, R. Millini, Chem. Soc. Rev., 2013, 42, 3956-3976.

[38] Q. Y. Wang, S. T. Xu, J. R. Chen, Y. X. Wei, J. Z. Li, D. Fan, Z. X. Yu, Y. Qi, Y. L. He, S. L. Xu, C. Y. Yuan, Y. Zhou, J. B. Wang, M. Z. Zhang, B. L. Su, Z. M. Liu, RSC Adv., 2014, 4, 21479-21491.

[39] L. T. Kong, Z. Jiang, J. G. Zhao, J. C. Liu, B. X. Shen, Catal. Lett., 2014, 144, 1609-1616.

[40] J. Ahmadpour, M. Taghizadeh, J. Nat. Gas Sci. Eng., 2015, 23, 184-194.

[41] C. Jo, J. Jung, H. S. Shin, J. Kim, R. Ryoo, Angew. Chem. Int. Ed., 2013, 52, 10014-10017.

[42] L. Xu, X. Y. Ji, S. H. Li, Z. Y. Zhou, X. Du, J. L. Sun, F. Deng, S. N. Che, P. Wu, Chem. Mater., 2016, 28, 4512-4521.

[43] M. Liu, W. Z. Jia, J. H. Li, Y. N. Wang, S. W. Ma, H. H. Chen, Z. R. Zhu, Catal. Lett., 2016, 146, 249-254.

[44] I. Majchrzak-Kucęba, W. Nowak, Int. J. Miner. Process., 2011, 101, 100-111.

[45] D. Zhao, C. Nie, Y. Zhou, S. Xia, L. Huang, Q. Li, Catal. Today, 2001, $68,11-20$.

[46] H. I. Meléndez-Ortiz, L. A. García-Cerda, Y. Olivares-Maldonado, G. Castruita, J. A. Mercado-Silva, Y. A. Perera-Mercado, Ceram. Int., 2012, 38, 6353-6358.

[47] Q. Cai, Z. S. Luo, W. Q. Pang, Y. W. Fan, X. H. Chen, F. Z. Cui, Chem. Mater., 2001, 13, 258-263.
[48] M. F. Ottaviani, A. Moscatelli, D. Desplantier-Giscard, F. Di Renzo, P. J. Kooyman, B. Alonso, A. Galarneau, J. Phys. Chem. B, 2004, 108, 12123-12129.

[49] Y. Jiang, Y. L. Wang, W. M. Zhao, J. Huang, Y. P. Zhao, G. S. Yang, Y. F. Lei, R. Z. Chu, J. Taiwan Inst. Chem. Eng., 2016, 61, 234-240.

[50] I. I. Ivanova, I. A. Kasyanov, A. A. Maerle, V. I. Zaikovskii, Microporous Mesoporous Mater., 2014, 189, 163-172.

[51] S. Y. Liu, J. Ren, H. K. Zhang, E. J. Lü, Y. Yang, Y. W. Li, J. Catal., 2016, $335,11-23$.

[52] W. Guo, L. Huang, P. Deng, Z. Xue, Q. Li, Microporous Mesoporous Mater., 2001, 44-45, 427-434.

[53] V. V. Ordomsky, I. I. Ivanova, E. E. Knyazeva, V. V. Yuschenko, V. I. Zaikovskii, J. Catal., 2012, 295, 207-216.

[54] C. T. Kresge, M. E. Leonowicz, W. J. Roth, J. C. Vartuli, J. S. Beck, Nature, 1992, 359, 710-712.

[55] Y. D. Xia, R. Mokaya, J. Mater. Chem., 2004, 14, 863-870.

[56] A. Karlsson, M. Stöcker, R. Schmidt, Microporous Mesoporous Mater., 1999, 27, 181-192.

[57] H. L. Chen, J. Ding, Y. M. Wang, New J. Chem., 2014, 38, 308-316.

[58] F. Hasan, R. Singh, G. Li, D. Y. Zhao, P. A. Webley, J. Colloid Interf. Sci., 2012, 382, 1-12.

[59] W. Tjandra, J. Yao, K. C. Tam, Langmuir, 2006, 22, 1493-1499.

[60] K. Egeblad, C. H. Christensen, M. Kustova, C. H. Christensen, Chem. Mater., 2008, 20, 946-960.

[61] L. L. Zhang, Y. Song, G. D. Li, S. L. Zhang, Y. S. Shang, Y. J. Gong, Acta Phys.-Chim. Sin., 2015, 31, 2139-2150.

[62] E. J. M. Hensen, D. G. Poduval, V. Degirmenci, D. A. J. M. Ligthart, W. B. Chen, F. Maugé, M. S. Rigutto, J. A. R. van Veen, J. Phys. Chem. C, 2012, 116, 21416-21429.

[63] Y. P. Khitev, Y. G. Kolyagin, I. I. Ivanova, O. A. Ponomareva, F. Thibault-Starzyk, J. P. Gilson, C. Fernandez, F. Fajula, Microporous Mesoporous Mater., 2011, 146, 201-207.

[64] S. Abelló, A. Bonilla, J. Pérez-Ramírez, Appl. Catal. A, 2009, 364, 191-198.

[65] P. Y. Dapsens, C. Mondelli, J. Perez-Ramirez, ChemSusChem, 2013, $6,831-839$.

\title{
利用双模板剂的多级孔ZSM-11分子篮的合成、表征和催化性能
}

\author{
刘 惠 ${ }^{\mathrm{a}, \mathrm{b},{ }^{*}}$, 张 爽, 谢素娟 ${ }^{\mathrm{a}}$, 张琬铩 ${ }^{\mathrm{a}, \mathrm{c}}$, 辛文杰 ${ }^{\mathrm{a}}$, 刘盛林 ${ }^{\mathrm{a}}$, 徐龙伢 ${ }^{\mathrm{a}, \#}$ \\ a 中国科学院大连化学物理研究所洁净能源国家实验室(筹), 辽宁大连 116023 \\ b 中化泉州石化有限公司研发中心, 福建泉州362103 \\ c哈尔滨师范大学化学化工学院, 黑龙江哈尔滨 150025
}

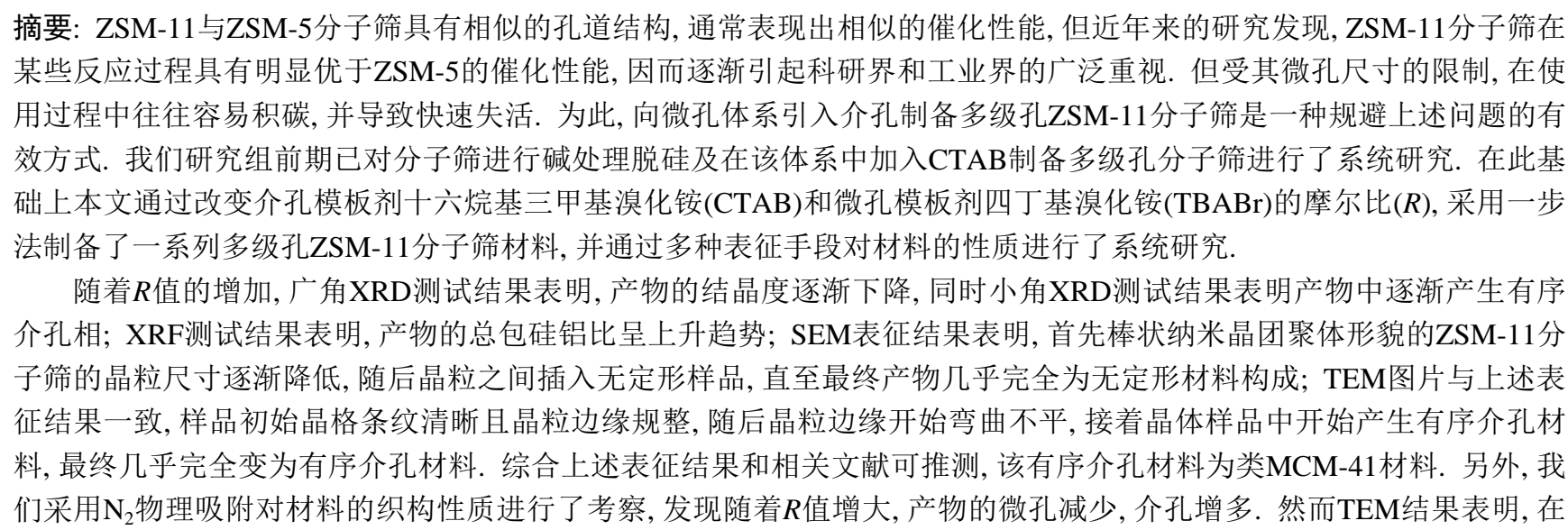


类MCM-41产生之前, 样品中介孔的增多并非来源于晶内孔, 而是来源于晶粒之间的堆积孔, 这是由于在合成过程中CTAB 胶束嵌入ZSM-11的晶粒之间所导致. 合成体系的碱度对产物的物化性质和织构性质也起了非常重要的作用. 我们将这个 体系与 $\mathrm{NaOH}+\mathrm{CTAB}$ 对分子篎进行脱硅后处理体系进行了对比, 发现虽然前者只能产生晶间孔而后者可产生晶内孔, 但碱 度对两者的作用相似, 即降低碱度均可导致类MCM-41有序介孔相的产生. 此外, 还通过 $\mathrm{NH}_{3}$-TPD和Py-IR技术对材料的酸 性进行了考察. 基于上述研究结果, 提出了多级孔ZSM-11分子笁的形成机理. 随着 $R$ 值的变化, CTAB分别起了覆盖效应、 胶束效应和模板效应. 这些效应在合成多级孔ZSM-11分子篮过程中相互协同, 同时又与TBABr的结构导向效应相互竞争, 进而影响合成产物的孔结构、形貌、酸性以及用于二甲醚和苯烷基化的催化性能.

关键词: 多级孔材料; ZSM-11; 双模板剂; 十六烷基三甲基澳化铵; 苯的烷基化

收稿日期: 2017-10-05. 接受日期: 2017-11-21. 出版日期: 2018-01-05.

*通讯联系人. 电话/传真: (0595)27570458; 电子信箱：liuhui22@sinochem.com

\#通讯联系人. 电话/传真: (0411)84379279; 电子信箱: lyxu@dicp.ac.cn

本文的电子版全文由Elsevier出版社在ScienceDirect上出版(http://www.sciencedirect.com/science/journal/18722067).

\section{《催化学报》为被国际期刊退稿的高质量论文开辟绿色通道}

我们注意到, 国内一些高质量、原创性的研究工作在投稿到国际期刊上时, 经常因语言和写作问题等非科 学因素而被退稿和拖延发表, 甚至会失去原创知识产权, 这个损失是巨大的, 也是非常可惜的!

众所周知, 按国际学术界的共识, 获得论文的首发权并保证拥有研究工作的原创性不在于论文发表在哪个 刊物(只要是国际ISSN挂号的), 而在于谁第一时间抢先发表了工作.

为了保护作者工作的原创性和首发知识产权, 《催化学报》编委会为具有一定原创性的论文提供快速发表 的绿色通道. 对于投到国际著名期刊(如Science, Nature, Energy \& Environmental Science, Journal of the American Chemical Society, Angewandte Chemie International Edition, ACS Catalysis和Journal of Catalysis等)上催化论 文的退稿, 如果其创新性较强, 学术质量较高, 仅仅是因为语言和文章写作原因而被退稿, 《催化学报》会安排 其在最近一期上抢时间发表; 如果文章原创性较好, 不存在学风问题, 但有一些理论观点的争议、实验现象暂 时无法得到完美的理论解释, 或者需要进一步补充相关实验数据等, 本刊主编、副主编也会协助作者尽快修改 和发表相关工作.

对于国际著名相关期刊的退稿, 作者需将相应的审稿意见及意见答复等材料一并提交到本刊, 以加快论文 的处理速度. 